OPEN ACCESS

Edited by:

Henry T. Nguyen,

University of Missouri, USA

Reviewed by:

Xiyin Wang,

North China University of Science and Technology, China

Swarup Kumar Parida,

National Institute of Plant Genome

Research, India

*Correspondence:

Oscar A. Ruiz

ruiz@intech.gov.ar

Specialty section:

This article was submitted to

Plant Genetics and Genomics,

a section of the journal

Frontiers in Plant Science

Received: 22 October 2015

Accepted: 11 March 2016

Published: 30 March 2016

Citation:

Calzadilla PI, Maiale SJ, Ruiz OA and

Escaray FJ (2016) Transcriptome

Response Mediated by Cold Stress in

Lotus japonicus.

Front. Plant Sci. 7:374.

doi: 10.3389/fp/s.2016.00374

\section{Transcriptome Response Mediated by Cold Stress in Lotus japonicus}

\author{
Pablo I. Calzadilla, Santiago J. Maiale, Oscar A. Ruiz * and Francisco J. Escaray \\ Unidad de Biotecnología 1, Instituto de Investigaciones Biotecnológicas, Instituto Tecnológico de Chascomús, Universidad \\ Nacional de San Martín, Consejo Nacional de Investigaciones Científicas y Técnicas, Chascomús, Argentina
}

Members of the Lotus genus are important as agricultural forage sources under marginal environmental conditions given their high nutritional value and tolerance of various abiotic stresses. However, their dry matter production is drastically reduced in cooler seasons, while their response to such conditions is not well studied. This paper analyzes cold acclimation of the genus by studying Lotus japonicus over a stress period of $24 \mathrm{~h}$. High-throughput RNA sequencing was used to identify and classify 1077 differentially expressed genes, of which 713 were up-regulated and 364 were down-regulated. Up-regulated genes were principally related to lipid, cell wall, phenylpropanoid, sugar, and proline regulation, while down-regulated genes affected the photosynthetic process and chloroplast development. Together, a total of 41 cold-inducible transcription factors were identified, including members of the AP2/ERF, NAC, MYB, and WRKY families; two of them were described as putative novel transcription factors. Finally, DREB1/CBFs were described with respect to their cold stress expression profiles. This is the first transcriptome profiling of the model legume $L$. japonicus under cold stress. Data obtained may be useful in identifying candidate genes for breeding modified species of forage legumes that more readily acclimate to low temperatures.

Keywords: legume, RNA-Seq, cold stress, transcriptome, forage

\section{INTRODUCTION}

Legumes (Fabaceae) are the primary source of plant protein for human consumption and in livestock feed, as well as key components of natural and agricultural ecosystems. Members of the Lotus genus, which includes about 120 species around the world, are particularly well known due to their elevated adaptability to marginal environmental conditions. This feature has made species of the Lotus genus popular as alternative forage in South America and Australia, as well as for dune revegetation and the reclamation of soil that has been burned or contaminated by heavy metals (Escaray et al., 2012). Within the genus, L. japonicus has become a model legume (Handberg and Stougaard, 1992), having been extensively used in abiotic stress (Díaz et al., 2010; Sainz et al., 2010; Babuin et al., 2014) and root nodulation studies (López et al., 2008; Li et al., 2014); this utility mainly results from particular genomic features that make it especially useful for recently developed functional genomics techniques (Sato et al., 2008; Fukai et al., 2012; Urbañski et al., 2012).

Even though forage Lotus species are considered cool season plants (Blumenthal and McGraw, 1999), dry matter production is in fact minimal during colder seasons at high latitudes (Bullard and Crawford, 1995; Halling et al., 2004), causing substantial economic losses (Wheeler et al., 2000; Thakur et al., 2010). It is therefore important for breeders to understand the mechanisms through which these plants tolerate cold and freezing. Previous research has focused on the cold 
stress response of Arabidopsis and has included both genetic and biochemical approaches (Mckown et al., 1996; Hannah et al., 2005; Kaplan et al., 2007). However, few studies have addressed legumes (Lucau-Danila et al., 2012; Dinari et al., 2013).

Plants have various ways in which they respond to and tolerate cold stress. These include changes in the composition, structure, and function of the plasma membrane, the synthesis of cryoprotectant molecules (soluble sugars and low-molecularweight nitrogenous compounds, such as proline), and an increase in the scavenging activity of reactive oxygen species (ROS; Steponkus, 1984; Apel and Hirt, 2004; Wang et al., 2013). Cold temperatures also induce distinct secondary metabolic pathways. In particular, flavonoids have been proposed as a primary target of study for understanding this phenomenon; this large family of metabolites, synthesized via the phenylpropanoid pathway, has been related to photoprotection, cold hardiness, drought resistance, and antioxidative ability (Christie et al., 1994; ChalkerScott, 1999).

Most cold stress responses are due to changes in gene expression, for which many transcription factors (TFs) have already been identified (Chinnusamy et al., 2007). The most well documented pathways involve a class of DREB/CBF TFs that are upstream regulated by several proteins, such as ICE1, MYB15, and ZAT12 (Shinozaki et al., 2003; Maruyama et al., 2009); the genes that are regulated through these pathways are collectively identified as cor genes. A large number of these genes encode proteins with known enzyme activity, specifically molecular chaperones and LEA proteins (Thomashow, 1999; Maruyama et al., 2009).

Next-generation sequencing has dramatically improved the efficiency of transcriptome data collection (Donà et al., 2013; Pang et al., 2013). High-throughput RNA sequencing (RNASeq) has been used in gene discovery and regulatory network studies, particularly in higher plants under stress (Deyholos, 2010; Kakumanu et al., 2012; Wang et al., 2013).

In this study, we describe the first transcriptome profiling by combining RNA-Seq with DREB1/CBF and cor gene expression analysis of the model legume L. japonicus after subjecting it to cold stress. To the best of our knowledge, this is the first report of $L$. japonicus response under low temperature stress. The results of this study can therefore guide the development of new agricultural forage practices and serve as a template for future studies that expand this understanding to other species within the genus.

\section{MATERIALS AND METHODS}

\section{Plant Materials and Growth Conditions}

Seeds from the L. japonicus ecotype Gifu B-129 were scarified by stirring in pure sulfuric acid for $3 \mathrm{~min}$, washed 10 times with sterile distilled water, and then sowed in Petri dishes containing $0.8 \%$ agar solution. Plates were incubated for 7 days in a growth chamber under a $16 / 8 \mathrm{~h}$ photoperiod at $24^{\circ} \mathrm{C} / 21^{\circ} \mathrm{C}$ $\pm 2{ }^{\circ} \mathrm{C}$ (day/night) and 55/65 $\pm 5 \%$ relative humidity. Light, at a photosynthetic flux density of $250 \mu \mathrm{mol} \mathrm{m} \mathrm{m}^{-2} \mathrm{~s}^{-1}$, was provided by $\mathrm{F}$ 40W Grolux fluorescent tubes. Seedlings were transferred to sterilized sand-perlite (2:1), placed in the previously used growth chamber under the same conditions, and irrigated with half-strenght Hoagland solution (Hoagland and Arnon, 1950).

Plants with 4-6 fully developed leaves, corresponding to roughly 3 weeks of development, were used in all experiments.

\section{Treatments and Experimental Design}

The experimental design was completely randomized, with three biological repetitions per treatment. For both the control and stress tests, the 3 week-old seedlings were placed in a Percival E-30B (Percival Scientific, Perry, IA, USA) growth chamber under a $16 / 8 \mathrm{~h}$ photoperiod (day/night). Lighting conditions were identical to those used in Section Plant Materials and Growth Conditions Plants under cold stress treatment were kept at $9^{\circ} \mathrm{C}$ during daylight simulation and $5^{\circ} \mathrm{C}$ at night, whereas the controls were kept at conditions identical to Section Plant Materials and Growth Conditions.

For RNA-Seq, shoots were harvested after 1 complete $24 \mathrm{~h}$ cycle, immediately frozen in liquid $\mathrm{N}_{2}$, and stored at $-80^{\circ} \mathrm{C}$ until their RNA was completely extracted. For DREB1/CBF and cor gene expression analysis, shoots were harvested after $0,1,3,8$, and $24 \mathrm{~h}$ of cold stress treatment.

\section{RNA Extraction and Expression Analysis}

Total RNA was extracted using a Plant Spectrum Total RNA Kit (Sigma) according to the manufacturer's instructions. RNA was checked for quality and quantified using agarose gel electrophoresis and spectrophotometric analysis. Total RNA samples $(5-10 \mu \mathrm{g} /$ sample) were shipped on dry ice to the Instituto de Agrobiotecnologia de Rosario (Rosario, Argentina) for RNA-Seq and analysis.

mRNA was purified using oligo-Dt and cDNA was synthesized. Samples were prepared for RNA sequencing as described in the Illumina TruSeq ${ }^{\circledR}$ RNA Sample Preparation Guide (July 2012). High-performance, paired-end $(2 \times 100$ bp) sequencing was performed on an Illumina Hiseq 1500. Low-quality RNA-Seq reads (Qscore < Q30) were analyzed using FastQC (Version 0.11.2) and discarded (Andrews, 2010). Then, a total of 174520000 reads were aligned against the $L$. japonicus genome (Gene Model, Release 2.5; Sato et al., 2008) using TopHat (Version 2.0.12; Trapnell et al., 2012).

To identify differentially expressed genes, a pair-wise comparison between normalized gene expression values (FPKM) for both conditions was performed using a $t$-test at the $99.99 \%$ confidence level using Cufflinks (Version 2.2.1; Trapnell et al., 2012). Illumina reads generated from all 6 samples are available at the NCBI BioProject browser, accession number PRJNA288510, BioSample accessions SAMN03801565, SAMN03801566, SAMN03801567, SAMN03801568, SAMN03801569, and SAMN03801570.

The overall RNA extraction procedure was identical for relative quantification of DREB1/CBF and cor genes. The absence of DNA from the RNA samples was tested by null polymerase chain reaction (PCR) amplification of the universal rDNA primer pair ITS1/ITS4 (Escaray et al., 2014). Then, cDNA was synthesized from $3 \mu \mathrm{g}$ of total RNA using M-MLV Reverse Transcriptase (Promega) and $100 \mathrm{pmol}$ of the oligo-Dt primers, as per the supplier's instructions. 


\section{RNA-Seq Transcript Analysis}

An MA-plot was constructed using R (Version 3.1.3) for Windows, which contained both the CummeRbund and ggplot2 packages (Team, 2014). All transcripts were used to make the plot.

Gene Ontology (GO) enrichment analysis was carried out to reveal the biological processes differentially expressed under the stress conditions, using Fisher's exact test against the L. japonicus entry in the legumeIP database (Al-Shahrour et al., 2004; Li et al., 2012). Blast2GO software (https://www.blast2go.com/) was used and applied only for those transcripts that showed significant differential expression, defined, with respect to fold change (FC), as those for which $\log _{2} \mathrm{FC} \geq|2|$ (Conesa et al., 2005).

Identification of the Kyoto Encyclopedia of Genes and Genomes (KEGG) metabolic pathways modified under low temperature treatment was used to corroborate the GO enrichment analysis (Kanehisa et al., 2010). KEGG annotation was done with the KEGG Automatic Annotation Server (http:// www.genome.jp/kaas-bin/kaas_main), again only for those transcripts for which $\log _{2} \mathrm{FC} \geq|2|$ (Moriya et al., 2007). Classification was also performed using the LegumeIP database (Li et al., 2012).

Up-regulated TFs were identified and classified through PlantTFDB (http://planttfdb.cbi.pku.edu.cn/) and the L. japonicus entry in the Legume IP database ( $\mathrm{Li}$ et al., 2012; Jin et al., 2014). Annotation information was obtained from GenBank (http://www.ncbi.nlm. nih.gov/genbank/, NCBI-GenBank Release 205.0, Dec, 2014www.ncbi.nlm.nih.gov/genbank/, NCBI-GenBank Release 205.0, Dec, 2014), LegumeIP [http://plantgrn.noble.org/ LegumeIP/, LegumeIP v2 (beta version)] and the KEGG database (http://www.genome.jp/kegg, Release 74.0, April 1, 2015; Kanehisa et al., 2010; Li et al., 2012).Transcripts not functionally annotated by one or more of the above methods were assigned functional annotations based on a BLASTX search vs. the Genbank NR protein database for $E$-values less than $10^{-3}$; the lowest $E$-value sequences were chosen as representative.

To validate the results obtained from RNA-Seq, the expression data of 10 randomly chosen genes were analyzed by quantitative real time PCR (qRT-PCR; Supplementary Table 1). A linear regression analysis of RNA-Seq and qRT-PCR values was completed, resulting in $R^{2}=0.71$ (Pearson's correlation $\mathrm{r}=0.84$; Supplementary Figure 1).

\section{qRT-PCR}

To validate the RNA-Seq analyses and to quantify the relative expression of DREB1/CBF and cor genes, primers were designed with the help of Primer3 software (Untergasser et al., 2012). The primer pairs are given in Supplementary Table 1. An aliquot of $5 \mu \mathrm{L}$ of $1: 8$ diluted cDNA was used in the qRTPCR reactions, made using $15 \mu \mathrm{L}$ of the FastStart Universal SYBR-Green Master Mix (Rox, Roche) and 2.5 pmol of each primer. Three biological replicates, every one accompanied by two technical replicates, were performed for each sample and gene. Cycling parameters were used that consisted of an initial step of $95^{\circ} \mathrm{C}$ for $10 \mathrm{~min}$ and a two-step cycle of $95^{\circ} \mathrm{C}$ for $30 \mathrm{~s}$ and $60^{\circ} \mathrm{C}$ for $1 \mathrm{~min}$, repeated 40 times. This was followed

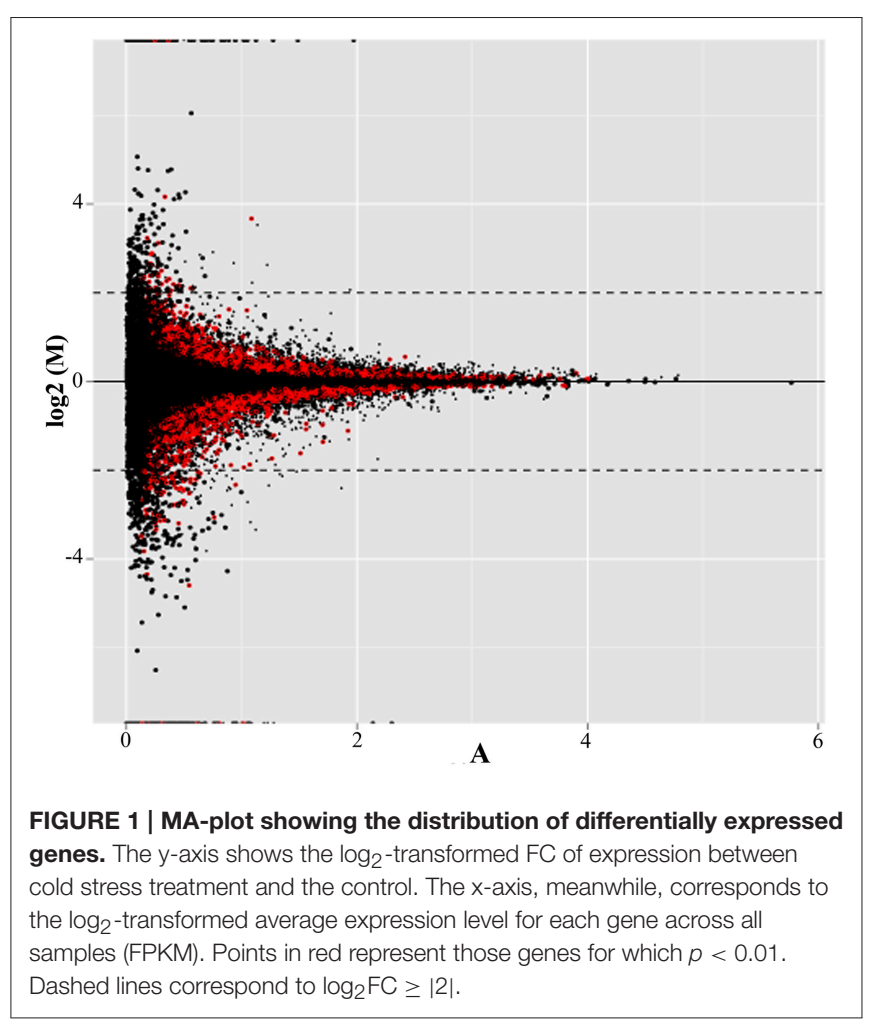

by the dissociation protocol. Amplifications were performed on an Mx3005P qPCR apparatus (Stratagene, CA, USA). The average threshold cycle was determined for each transcript. Gene quantification was determined based on the relative expression of the target gene vs. the reference gene EF-1 $\alpha$ (Escaray et al., 2014). For comparative purposes, average threshold cycle values of the control samples were used as reference.

The statistical analysis of relative gene expression was performed using the InfoStat/L program (Di Rienzo et al., 2008).

\section{RESULTS}

\section{Sequencing and Read Assembly}

Almost $68 \%$ of the transcripts resulting from read alignment mapped with the reference genome, allowing for the identification of 40993 genes (Figure 1). In total, 9781, or approximately $24 \%$, were differentially expressed between treatments $(p<0.01$, represented as red points in Figure 1). Of those genes, 1077 , or about $11 \%$, showed more than a two-fold change in their expression ratio. Within this group, 713 genes were up-regulated under cold stress treatment, while 364 were down-regulated; 133 and 75 of these genes, respectively, have no annotation in the L. japonicus genome. These 1077 genes were used for functional classification and annotation. It is worth noting that the percentage of mapped reads obtained was expected due to unavailability of complete genome information and the presence of highly repetitive sequences in the L. japonicus genome (Sato and Andersen, 2014). 


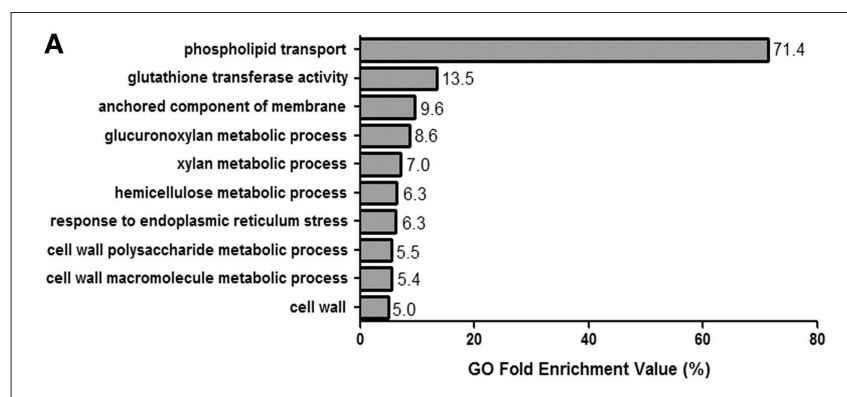

B

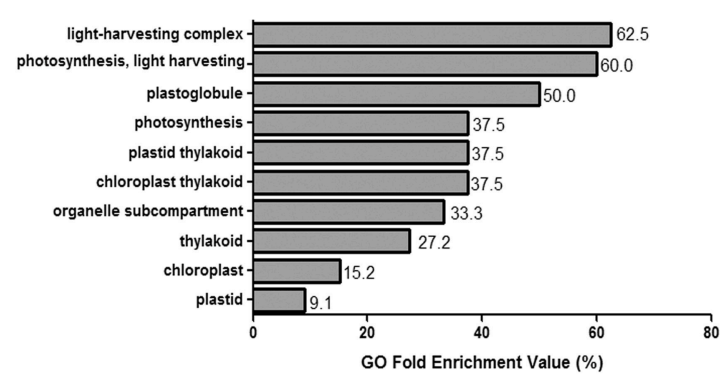

FIGURE 2 | GO enrichment analysis.(A,B) The 10 GO terms that are most representative for each set of the 713 up-regulated and 364 down-regulated genes, as defined by those for which $\log _{2} F C \geq 2$ and $\log _{2} F C \geq-2$, respectively. GO enrichment values (represented as percentages) were calculated by generating the ratios for the number of observed over expected GO term repeats.

\section{Functional Annotation and Classification GO Enrichment Analysis}

GO terms were successfully identified for $87.2 \%$ of the transcripts. The ten GO terms most highly represented in the upand down-regulated gene list, with false discovery rate $p$-values smaller than or equal to 0.01 , were identified (Figures 2A,B, respectively).

The main up-regulated GO term was phospholipid transport, followed by features related to plasma membrane composition, specifically with respect to the anchored component of the membrane and response to endoplasmic reticulum stress. In addition, six of the ten up-regulated GO terms were related to the cell wall, both specifically to the cell wall and to the glucuronoxylan, xylan, hemicellulose, cell wall polysaccharide, and cell wall macromolecule metabolic processes. The gluthatione transferase activity GO term, related generally to glutathione metabolism, was also highly up-regulated. Meanwhile, the light harvesting complex, light harvesting, and photosynthesis GO terms were significantly down-regulated.

\section{KEGG Metabolic Pathway Identification}

A total of $579(66.6 \%)$ of the annotated genes matched a KEGG pathway, with the highest percentages pertaining to the metabolism, organismal system, and human diseases categories for both the up- and down-regulated genes (Figures 3A,B, respectively). After metabolism, organismal system was the most represented category in the KEGG analysis (Figure 3). Many of the up-regulated genes in this category were classified as

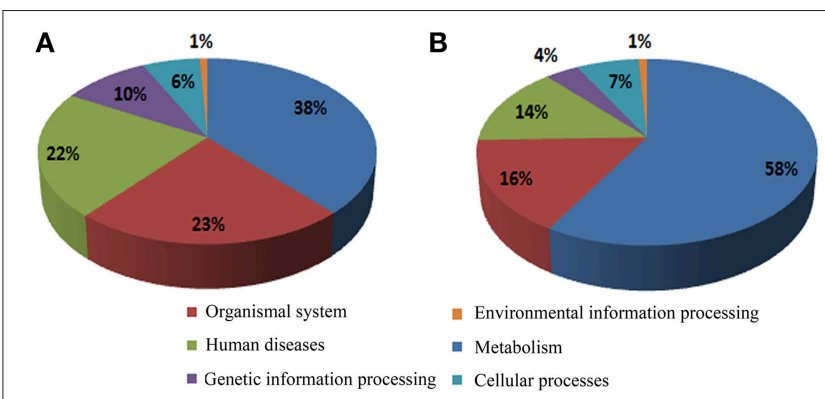

FIGURE 3 | Assignment of cold stress responsive genes to the KEGG Pathway Database. (A,B) Classification of up- and down-regulated genes, respectively.

heat shock protein 1/8 (K03283) and interleukin-1 receptorassociated kinase 4 [EC:2.7.11.1] (K04733) (Supplementary Table 2). Unexpectedly, the least represented category in both cases was that of environmental information processing. However, some TFs were identified in the environmental adaptation pathway (Supplementary Table 2); almost all of them were up-regulated.

The different genes were then further classified within these categories (Figure 4); it is worth noting that within the organismal system classification, environmental adaptation was most heavily represented, with 44 genes significantly affected. Overall, the KEGG results support conclusions drawn from the GO data. Glutathione S-transferase genes were up-regulated (Table 1), as were most of those for starch and sucrose metabolism. In addition, the transcription of some of the genes involved in arginine and proline metabolism were upregulated by more than a factor of two. Meanwhile, lightharvesting complex proteins were down-regulated, as were the chloroplast and thylakoid membrane terms, including those of the plastoglobule, plastid thylakoid, and chloroplast thylakoid. Finally, cutin, suberine, and wax biosyntheses in the lipid metabolism category were down-regulated.

Furthermore, the genes involved in the biosynthesis of secondary metabolites pathway in the KEGG metabolism category were over-represented; phenylpropanoid biosynthesis and flavonoid pathways were especially notable, with the majority of the genes up-regulated in the phenylpropanoid pathway being peroxidases [EC:1.11.1.7] (Table 1). In addition, one CHS ortholog (chr2.CM0018.1130.r2.m) and two CHI orthologs (chr5.CM0180.660.r2.m and chr5.CM0180.670.r2.m) were up-regulated. Finally, RNA-Seq demonstrated a downregulation of genes related to brassinosteroid (BR) biosynthesis (included under metabolism of terpenoids) and steroid hormone biosynthesis (included under lipid metabolism).

\section{TF Identification and Classification}

The expression levels of 41 plant TFs were up-regulated by low temperature. These TFs were sorted into 14 different families (Figure 5), with seven of these classified as MYB TFs; one of these (transcript XLOC_019119) was one of the two genes that had not been previously annotated (Supplementary Table 3). Additionally, five of the up-regulated TFs belonged to 


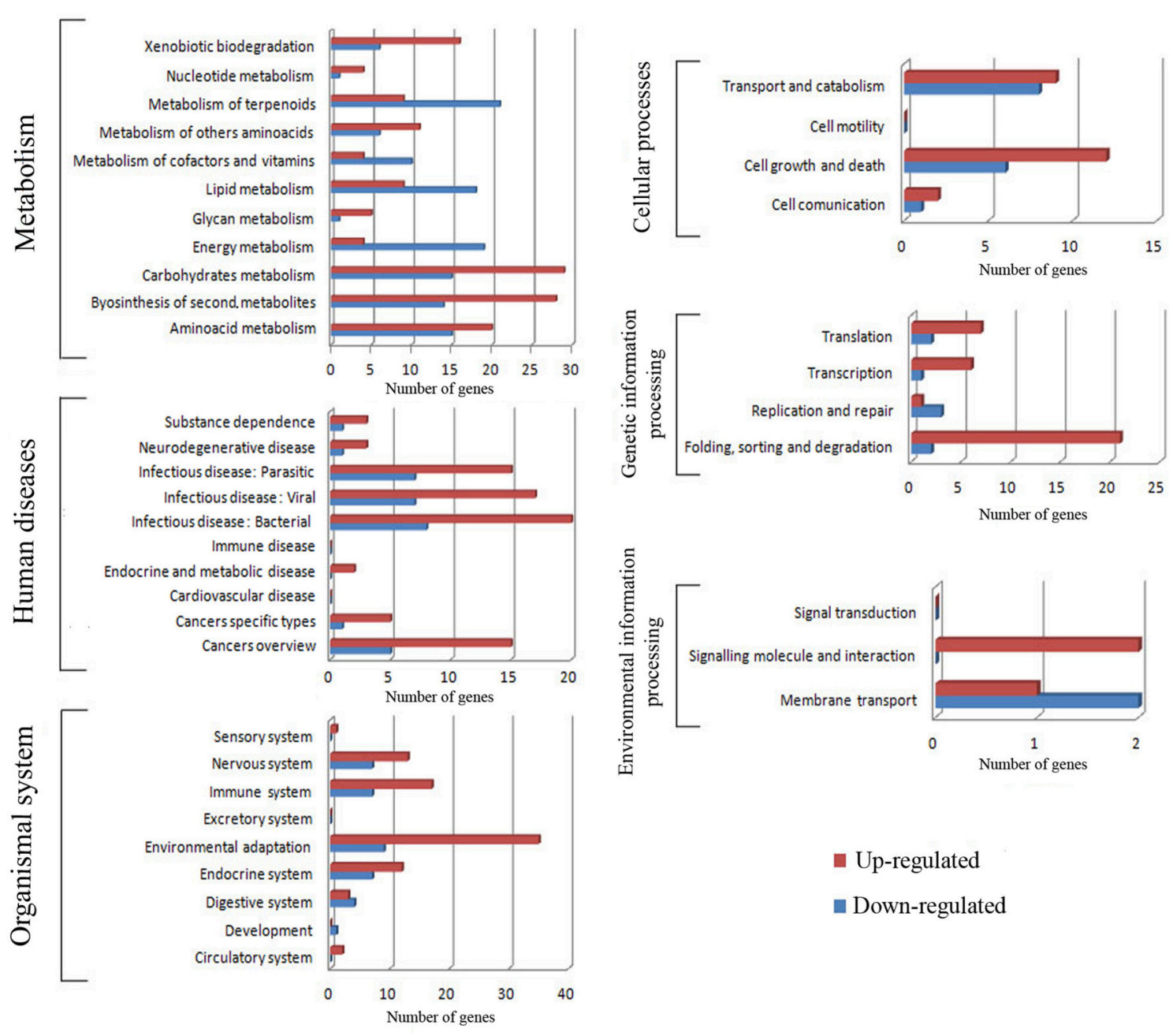

FIGURE 4 | Pathway subclassification for cold stress responsive genes for each KEGG category.

the NAC family, while six were part of the AP2/ERF family. One calmodulin binding transcription activator (CAMTA) TF (chr4.CM0307.390.r2.d) was found up-regulated, along with a number of calmodulin genes (Supplementary Tables 2, 3).

\section{Differentially Expressed Genes with no Previous Annotation}

Overall, $50(37.6 \%)$ and $24(32.0 \%)$ of the up- and downregulated genes, respectively, did not hit any sequence in the nonredundant protein GenBank database. Meanwhile, 27 (20.3\%) and 20 (26.7\%) of the up- and down-regulated genes, respectively, hit for an E-value greater than $10^{-3}$. The rest of the transcripts matched a sequence with an E-value lower than $10^{-3}$; however, from those, $22(39.3 \%)$ and $10(24.4 \%)$ of the up- and downregulated genes, respectively, hit to an unknown protein. In all, 34 $(60.7 \%)$ and 21 (75.6\%) of the remaining up- and down-regulated transcripts, respectively, matched with a characterized sequence in the GenBank database (Supplementary Tables 4, 5).
Aside from the two previously discussed, non-annotated transcripts that were identified as TFs (Supplementary Table 3 ), other up-regulated sequences gave transcripts that hit with ribonucleases, retrotransposon proteins, serine/threonineprotein kinases, a sucrose-phosphate synthase 4, fatty acid protein elongation, and a cellulose synthase-like protein (Supplementary Table 4). Meanwhile, the down-regulated genes contained transcripts that matched with the ribonuclease $\mathrm{H}$ family, a serine/threonine protein kinase, a mitochondrial arginine transporter, and a nuclear pore complex protein (Supplementary Table 5).

\section{DREB1/CBF Gene Expression Profiling}

After $24 \mathrm{~h}$ of cold stress, L. japonicus showed no expression differences in this family of regulatory genes, though their response may be evident at shorter times. For this reason, relative expression profiles were studied.

Measurements showed a peak of CBF1 (chr4.CM0126.2110.r2.a) and CBF3 (chr4.CM0126.2020.r2.a) 
TABLE 1 | Differentially expressed genes classified under the metabolism category of the KEGG Pathway Database.

\begin{tabular}{|c|c|c|c|c|c|c|}
\hline $\begin{array}{l}\text { KEGG pathway DB } \\
\text { metabolism }\end{array}$ & & Gene accession & $\log _{2} F C$ & $p$-value & KEGG annotation & ко \\
\hline \multirow[t]{6}{*}{ Aminoacid metabolism } & $\begin{array}{l}\text { Arginine and proline } \\
\text { metabolism }\end{array}$ & LjSGA_056129.1 & 2.10 & $1.00 \mathrm{E}-04$ & $\begin{array}{l}\text { Proline dehydrogenase } \\
\text { [EC:1.5.-.-] }\end{array}$ & K00318 \\
\hline & & LjSGA_006172.2 & 2.23 & $5.00 E-05$ & $\begin{array}{l}\text { Delta-1-pyrroline-5-carboxylate } \\
\text { synthetase }[E C: 2 \cdot 7.2 \cdot 11 \text { 1.2.1.41] }\end{array}$ & K12657 \\
\hline & & LjSGA_032463.1 & 2.06 & $5.00 \mathrm{E}-05$ & $\begin{array}{l}\text { Delta-1-pyrroline-5-carboxylate } \\
\text { synthetase [EC:2.7.2.11 1.2.1.41] }\end{array}$ & K12657 \\
\hline & & chr3.CM0711.100.r2.d & -2.44 & $5.00 \mathrm{E}-05$ & $\begin{array}{l}\text { Agmatine coumaroyltransferase } \\
\text { [EC:2.3.1.64 2.3.1.-] }\end{array}$ & K14329 \\
\hline & & chr3.CM0711.120.r2.d & -2.10 & $5.00 E-05$ & $\begin{array}{l}\text { Agmatine coumaroyltransferase } \\
\text { [EC:2.3.1.64 2.3.1.-] }\end{array}$ & K14329 \\
\hline & & chr6.LjT4OF03.150.r2.d & -2.50 & $5.00 \mathrm{E}-05$ & $\begin{array}{l}\text { Agmatine coumaroyltransferase } \\
\text { [EC:2.3.1.64 2.3.1.-] }\end{array}$ & K14329 \\
\hline & & chr6.CM0314.450.r2.m & -2.50 & $5.00 \mathrm{E}-05$ & $\begin{array}{l}\text { Anthocyanidin 3-O-glucoside } \\
\text { 2"'-O-xylosyltransferase } \\
\text { [EC:2.4.2.51] }\end{array}$ & K17193 \\
\hline & & LjSGA_028050.1 & -2.31 & $5.00 \mathrm{E}-05$ & $\begin{array}{l}\text { Anthocyanidin 3-O-glucoside } \\
\text { 2"'-O-xylosyltransferase } \\
\text { [EC:2.4.2.51] }\end{array}$ & K17193 \\
\hline & \multirow[t]{7}{*}{$\begin{array}{l}\text { Flavonoid } \\
\text { biosynthesis }\end{array}$} & chr2.CM0191.680.r2.m & 2.11 & $5.00 E-05$ & $\begin{array}{l}\text { Flavonol 3-O-methyltransferase } \\
\text { [EC:2.1.1.76] }\end{array}$ & K05279 \\
\hline & & CM1092.70.r2.m & 2.35 & $5.00 \mathrm{E}-05$ & Polyketide reductase & K08243 \\
\hline & & chr2.CM0124.1170.r2.d & 4.77 & $5.00 \mathrm{E}-05$ & $\begin{array}{l}\text { Leucoanthocyanidin dioxygenase } \\
\text { [EC:1.14.11.19] }\end{array}$ & K05277 \\
\hline & & chr5.CM0148.40.r2.m & 3.33 & $5.00 \mathrm{E}-05$ & $\begin{array}{l}\text { Shikimate } \\
\text { O-hydroxycinnamoyltransferase } \\
\text { [EC:2.3.1.133] }\end{array}$ & K13065 \\
\hline & & chr2.CM0002.140.r2.m & -2.27 & $5.00 \mathrm{E}-05$ & Flavonol synthase [EC:1.14.11.23] & K05278 \\
\hline & & chr3.CM0590.840.r2.m & -2.71 & $5.00 \mathrm{E}-05$ & Chalcone synthase [EC:2.3.1.74] & K00660 \\
\hline & & LjSGA_035363.1 & -2.47 & $1.00 \mathrm{E}-04$ & $\begin{array}{l}\text { Coumaroylquinate(coumaroylshikimate) } \\
\text { 3'-monooxygenase } \\
\text { [EC:1.14.13.36] }\end{array}$ & K09754 \\
\hline & \multirow[t]{11}{*}{$\begin{array}{l}\text { Phenylpropanoid } \\
\text { biosynthesis }\end{array}$} & chr1.CM0221.250.r2.d & 5.13 & $5.00 \mathrm{E}-05$ & Peroxidase [EC:1.11.1.7] & K00430 \\
\hline & & chr2.CM1032.300.r2.m & 2.79 & $5.00 \mathrm{E}-05$ & Peroxidase [EC:1.11.1.7] & K00430 \\
\hline & & chr2.CM0056.580.r2.d & 2.39 & $5.00 \mathrm{E}-05$ & Beta-glucosidase [EC:3.2.1.21 & K01188 \\
\hline & & chr6.CM0118.420.r2.m & 2.68 & $5.00 \mathrm{E}-05$ & Beta-glucosidase [EC:3.2.1.21] & K01188 \\
\hline & & chr6.CM0314.280.r2.m & 2.16 & $6.85 \mathrm{E}-03$ & Peroxidase [EC:1.11.1.7] & K00430 \\
\hline & & chr6.CM0314.270.r2.m & 2.04 & $5.00 \mathrm{E}-05$ & Peroxidase [EC:1.11.1.7] & K00430 \\
\hline & & LjSGA_014865.1 & 2.93 & $2.00 \mathrm{E}-04$ & Peroxidase [EC:1.11.1.7] & K00430 \\
\hline & & LjSGA_027137.1 & 2.63 & $1.33 E-02$ & Peroxidase [EC:1.11.1.7] & K00430 \\
\hline & & LjSGA_036806.1 & 2.31 & $5.00 \mathrm{E}-05$ & Peroxidase [EC:1.11.1.7] & K00430 \\
\hline & & LjSGA_043353.1 & 2.85 & $5.00 \mathrm{E}-05$ & Peroxidase [EC:1.11.1.7] & K00430 \\
\hline & & LjSGA_133676.2.1 & 2.27 & $5.00 \mathrm{E}-05$ & Peroxidase [EC:1.11.1.7] & K00430 \\
\hline
\end{tabular}


TABLE 1 | Continued.

\begin{tabular}{|c|c|c|c|c|c|c|}
\hline \multirow[t]{5}{*}{$\begin{array}{l}\text { KEGG pathway DB } \\
\text { metabolism }\end{array}$} & & Gene accession & $\log _{2} \mathrm{FC}$ & $p$-value & KEGG annotation & ко \\
\hline & & chr3.CM0241.540.r2.d & -2.82 & 5.00E-05 & $\begin{array}{l}\text { Ferulate-5-hydroxylase } \\
\text { [EC:1.14.-.-] }\end{array}$ & K09755 \\
\hline & & chr4.CM0046.1730.r2.m & -2.28 & $5.00 \mathrm{E}-05$ & Beta-glucosidase [EC:3.2.1.21] & K01188 \\
\hline & & chr5.CM0077.770.r2.d & -2.77 & $5.00 \mathrm{E}-05$ & Peroxidase [EC:1.11.1.7] & K00430 \\
\hline & & chr5.CM1324.140.r2.d & -3.33 & $5.00 \mathrm{E}-05$ & Beta-glucosidase [EC:3.2.1.21] & K01188 \\
\hline \multirow[t]{16}{*}{ Carbohydrate metabolism } & $\begin{array}{l}\text { Starch and sucrose } \\
\text { metabolism }\end{array}$ & LjT44L11.110.r2.d & 3.52 & 4.00E-04 & Pectinesterase [EC:3.1.1.11] & K01051 \\
\hline & & chr1.CM0122.2580.r2.m & 3.45 & $5.00 \mathrm{E}-05$ & Pectinesterase [EC:3.1.1.11] & K01051 \\
\hline & & chr2.CM0191.440.r2.m & 2.28 & $8.50 \mathrm{E}-04$ & $\begin{array}{l}\text { Galacturan } \\
\text { 1.4-alpha-galacturonidase } \\
\text { [EC:3.2.1.67] }\end{array}$ & K01213 \\
\hline & & chr1.CM0122.2540.r2.m & 2.11 & $5.00 \mathrm{E}-05$ & Sucrose synthase [EC:2.4.1.13] & K00695 \\
\hline & & chr2.CM0021.1140.r2.a & 3.63 & $5.00 \mathrm{E}-05$ & Beta-amylase [EC:3.2.1.2] & K01177 \\
\hline & & chr2.CM0056.580.r2.d & 2.39 & $5.00 \mathrm{E}-05$ & Beta-glucosidase [EC:3.2.1.21] & K01188 \\
\hline & & chr4.CM0004.1850.r2.a & 2.04 & $5.00 \mathrm{E}-05$ & $\begin{array}{l}\text { Alpha.alpha-trehalase } \\
\text { [EC:3.2.1.28] }\end{array}$ & K01194 \\
\hline & & chr6.CM0118.420.r2.m & 2.68 & $5.00 \mathrm{E}-05$ & Beta-glucosidase [EC:3.2.1.21] & K01188 \\
\hline & & LjSGA_011684.1 & 2.25 & 5.00E-05 & $\begin{array}{l}\text { Glucan 1.3-beta-glucosidase } \\
\text { [EC:3.2.1.58] }\end{array}$ & K01210 \\
\hline & & LjSGA_032594.1 & 2.33 & $5.00 \mathrm{E}-05$ & $\begin{array}{l}\text { Glucan 1.3-beta-glucosidase } \\
\text { [EC:3.2.1.58] }\end{array}$ & K01210 \\
\hline & & chr2.CM0081.1340.r2.m & 2.09 & 5.00E-05 & $\begin{array}{l}\text { Alpha-1.4- } \\
\text { galacturonosyltransferase } \\
\text { [EC:2.4.1.43] }\end{array}$ & K13648 \\
\hline & & chr4.CM0046.1730.r2.m & -2.28 & $5.00 \mathrm{E}-05$ & Beta-glucosidase [EC:3.2.1.21] & K01188 \\
\hline & & chr5.CM1324.140.r2.d & -3.33 & $5.00 \mathrm{E}-05$ & Beta-glucosidase [EC:3.2.1.21] & K01188 \\
\hline & & LjSGA_051150.1 & -2.26 & 5.00E-05 & Beta-glucosidase [EC:3.2.1.21] & K01188 \\
\hline & & chr3.CM0091.1230.r2.d & -2.16 & $5.00 \mathrm{E}-05$ & $\begin{array}{l}\text { Glucose-1-phosphate } \\
\text { adenylyltransferase [EC:2.7.7.27] }\end{array}$ & K00975 \\
\hline & & LjSGA_114621.1 & -2.13 & $5.00 \mathrm{E}-05$ & $\begin{array}{l}\text { Alpha-1.4- } \\
\text { galacturonosyltransferase } \\
\text { [EC:2.4.1.43] }\end{array}$ & K13648 \\
\hline \multirow[t]{6}{*}{ Energy metabolism } & $\begin{array}{l}\text { Photosynthesis - } \\
\text { antenna proteins }\end{array}$ & LjT24M21.140.r2.a & -2.73 & $5.00 \mathrm{E}-05$ & $\begin{array}{l}\text { Light-harvesting complex II } \\
\text { chlorophyll a/b binding protein } 4\end{array}$ & K08915 \\
\hline & & chr6.CM0314.310.r2.m & -2.48 & $5.00 \mathrm{E}-05$ & $\begin{array}{l}\text { Light-harvesting complex II } \\
\text { chlorophyll a/b binding protein } 6\end{array}$ & K08917 \\
\hline & & LjSGA_008354.1 & -2.97 & 5.00E-05 & $\begin{array}{l}\text { Light-harvesting complex I } \\
\text { chlorophyll a/b binding protein } 1\end{array}$ & K08907 \\
\hline & & LjSGA_008355.1 & -2.87 & 5.00E-05 & $\begin{array}{l}\text { Light-harvesting complex I } \\
\text { chlorophyll a/b binding protein } 1\end{array}$ & K08907 \\
\hline & & LjSGA_028870.1 & -2.40 & 5.00E-05 & $\begin{array}{l}\text { Light-harvesting complex I } \\
\text { chlorophyll a/b binding protein } 2\end{array}$ & K08908 \\
\hline & & LjSGA_036743.1 & -2.41 & 5.00E-05 & $\begin{array}{l}\text { Light-harvesting complex I } \\
\text { chlorophyll a/b binding protein } 2\end{array}$ & K08908 \\
\hline
\end{tabular}


TABLE 1 | Continued.

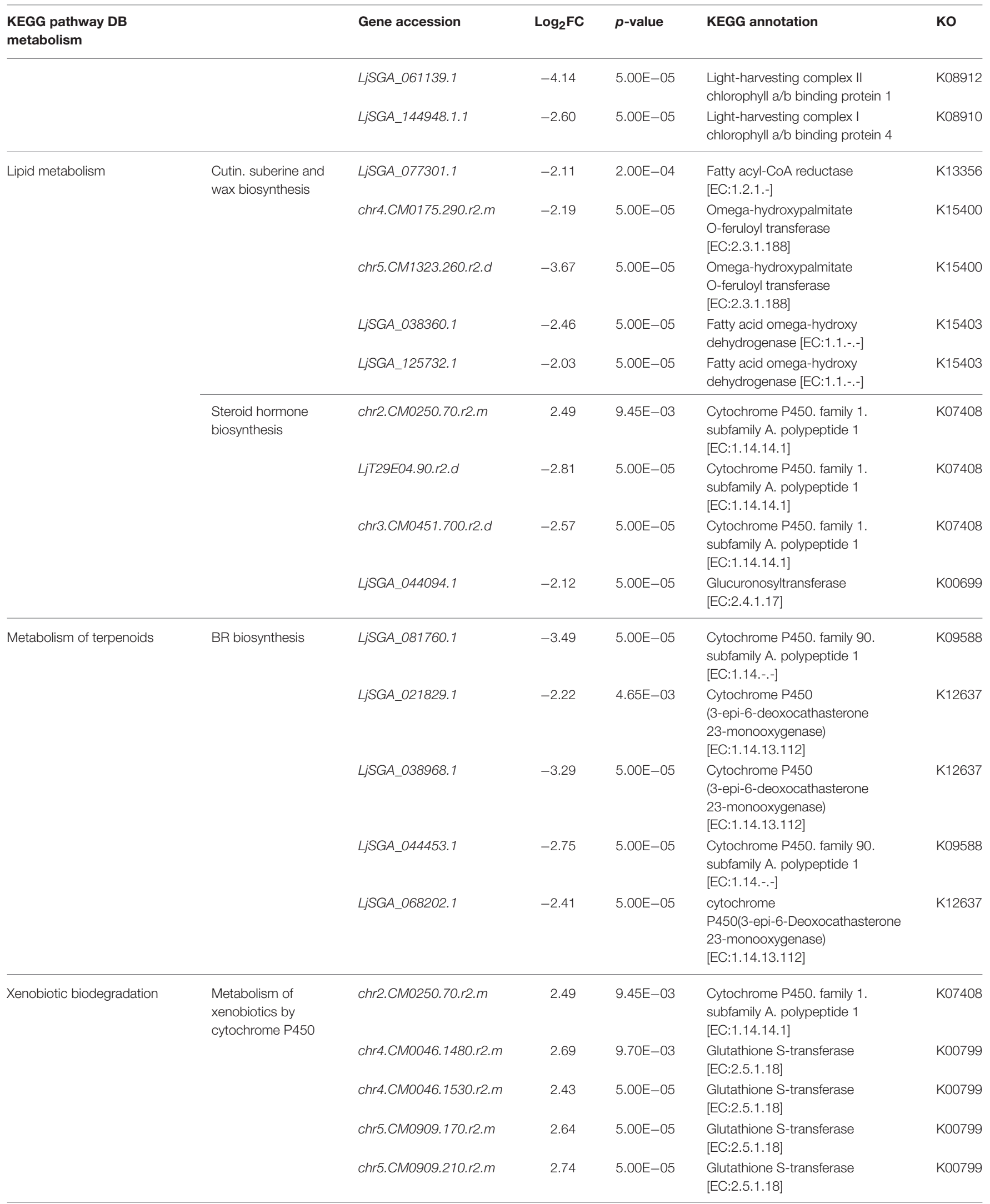


TABLE 1 | Continued.

\begin{tabular}{|c|c|c|c|c|c|}
\hline $\begin{array}{l}\text { KEGG pathway DB } \\
\text { metabolism }\end{array}$ & Gene accession & $\log _{2} F C$ & $p$-value & KEGG annotation & KO \\
\hline & LjSGA_018510.1 & 3.90 & $5.00 \mathrm{E}-05$ & $\begin{array}{l}\text { Glutathione S-transferase } \\
\text { [EC:2.5.1.18] }\end{array}$ & K00799 \\
\hline & LjSGA_030344.1 & 3.60 & $5.00 \mathrm{E}-05$ & $\begin{array}{l}\text { Glutathione S-transferase } \\
\text { [EC:2.5.1.18] }\end{array}$ & K00799 \\
\hline & LjSGA_055009.1 & 2.02 & $5.00 \mathrm{E}-05$ & $\begin{array}{l}\text { Glutathione S-transferase } \\
\text { [EC:2.5.1.18] }\end{array}$ & K00799 \\
\hline & chr3.CM0451.700.r2.d & -2.57 & $5.00 \mathrm{E}-05$ & $\begin{array}{l}\text { Cytochrome P450. family } 1 . \\
\text { subfamily A. polypeptide } 1 \\
\text { [EC:1.14.14.1] }\end{array}$ & K07408 \\
\hline & LjSGA_044094.1 & -2.12 & $5.00 \mathrm{E}-05$ & $\begin{array}{l}\text { Glucuronosyltransferase } \\
\text { [EC:2.4.1.17] }\end{array}$ & K00699 \\
\hline
\end{tabular}

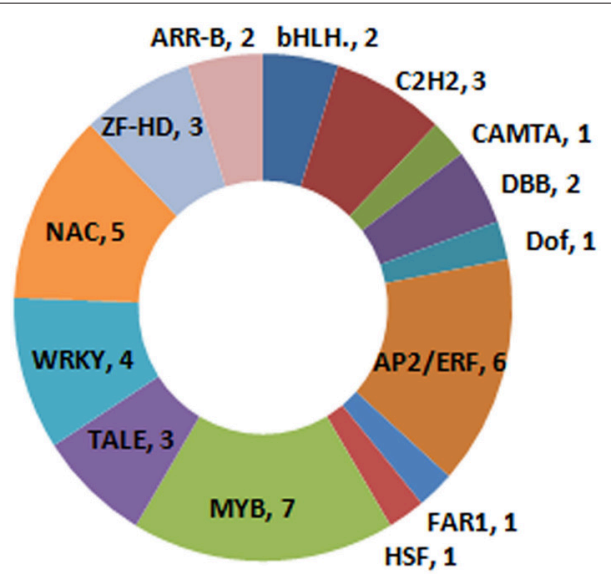

FIGURE 5 | Classification of cold stress up-regulated TFs.

ortholog expression after $1 \mathrm{~h}$ of treatment (Figures 6A,B), though these expression levels dropped as time went on. Nonetheless, after $24 \mathrm{~h}, \mathrm{CBF} 1$ expression was still significantly higher than the control conditions, even while CBF3 was no longer overexpressed. On the other hand, the relative expression pattern of the CBF2 (chr5.CM0359.290.r2.m) ortholog decreased after $1 \mathrm{~h}$ of stress and remained low throughout the treatment (Figure 6C). Meanwhile, ZAT12 (chr5.CM0180.280.r2.m) ortholog expression showed no significant changes (Figure 6D), even while the ICE1 (chr1.CM0104.550.r2.m) ortholog was down-regulated (Figure 6E). Finally, RD29A (chr5.CM0148.540.r2.m) and COR47 (chr1.CM0113.680.r2.d), two DREB1/CBF TF target genes, showed the highest expression after $8 \mathrm{~h}$, although this increase in expression became evident for RD29A after just $8 \mathrm{~h}$ of treatment (Figures 6F,G, respectively).

\section{DISCUSSION}

Because metabolism was the most expressed category in the KEGG classification, we further analyzed these pathways. The organismal system category, the second most expressed, was also analyzed.

\section{Metabolism Category Lipid Metabolism}

The regulation results help to account for observations related to lipid metabolism in previous studies. With respect to upregulation, earlier work had demonstrated molecular changes in the lipid membrane of plants undergoing cold and freezing stress (Iba, 2002; Welti et al., 2002) due to altered lipid biosynthesis and biomembrane rearrangement (Smolenska and Kuiper, 1977), as well as specific fatty acid changes (Willemot et al., 1977). Meanwhile, with respect to down-regulation, plants have been shown to decrease their cuticular wax content when subjected to cold stress (Shepherd and Wynne Griffiths, 2006), which is consistent not just with these results but with the negative correlation observed between frost tolerance and high epicuticular wax load in Salix sp. (Hietala et al., 1997).

Changes in the composition of wax due to stress must also be analyzed, as these features play a major role in stressor response; for example, two transgenic Arabidopsis plants with significantly enhanced drought tolerance were shown to differ in both wax composition and differential freezing response (Zhang et al., 2005, 2007).

\section{Cell Wall Metabolism}

Genes related to the cell wall are regulated by many different abiotic stresses, particularly water deficit stress and wounding; during severe low temperature conditions, extracellular ice formation could result in cell dehydration, collapse, or lysis due to ice crystal extension (Gall et al., 2015). This explains the strong up-regulation of genes involved in cell wall modification during low temperature stress, both in the literature (Domon et al., 2013; Dong et al., 2013) and in this study.

\section{Xenobiotic Biodegradation}

Many authors have highlighted the important role of diverse gluthatione-dependent enzymes in detoxification of xenobiotics and ROS, as well as in the adaptation to different abiotic 


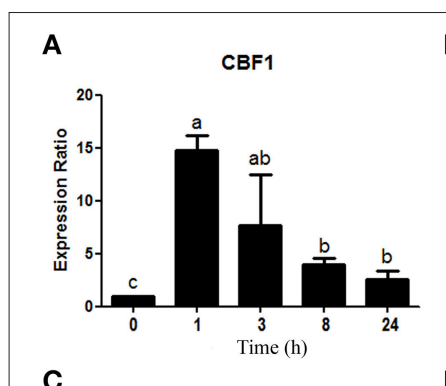

C

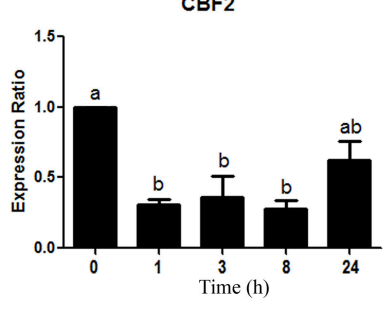

E

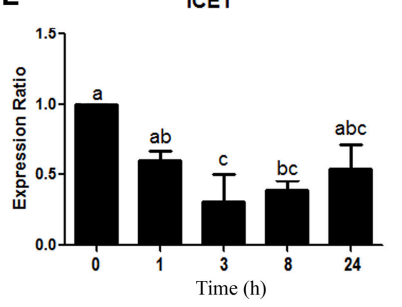

G

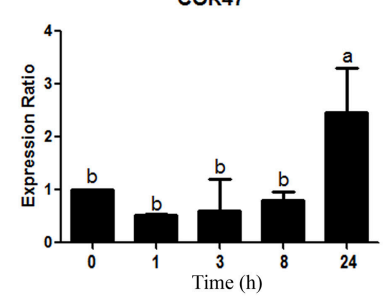

B

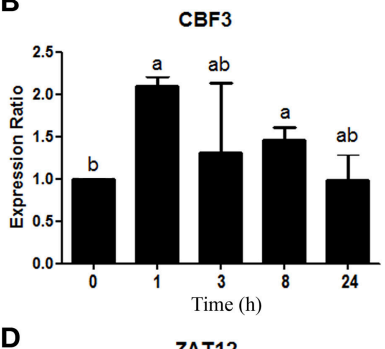

D

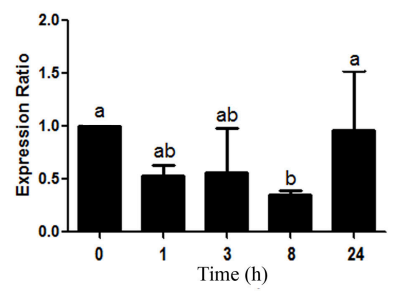

$\mathbf{F}$

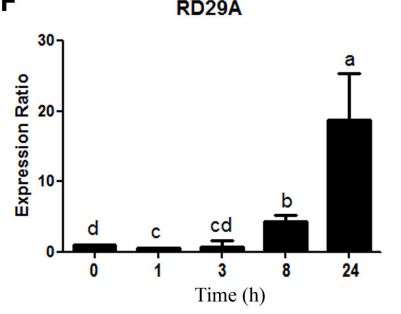

FIGURE 6 | Expression analysis of 7 genes involved in the DREB1/CBF regulon, according to qRT-PCR. Data was obtained at $0,1,3,8$, and $24 \mathrm{~h}$ after cold stress imposition. Expression analysis of the (A) CBF1 (chr4.CM0126.2110.r2.a), (B) CBF3 (chr4.CM0126.2020.r2.a), (C) CBF2 (chr5.CM0359.290.r2.m), (D) ZAT12 (chr5.CM0180.280.r2.m), (E) ICE1 (chr1.CM0104.550.r2.m), (F) RD29A (chr5.CM0148.540.r2.m), and (G) COR47 (chr1.CM0113.680.r2.d) orthologs. Values represent the mean $(n=3)$ \pm SEM. Means labeled with the same letters do not differ significantly

$(p<0.05)$, while bars indicate standard error of the mean values.

stresses, particularly cold stress (Dixon et al., 1998; Anderson and Davis, 2004; Halusková et al., 2009). During cooling, $\mathrm{H}_{2} \mathrm{O}_{2}$ and other ROS may accumulate, causing oxidative stress and cellular damage. In these conditions, reduced glutathione could first be oxidized by ROS, then regenerated by the activity of NADPHdependent gluthatione reductase [EC:1.6.4.2]. Unsurprisingly, enhanced glutathione content and glutathione reductase activity have been correlated to cold stress in different plant species (Kocsy et al., 2001). In fact, overexpressing gluthatione Stransferase [EC:2.5.1.18] in transgenic rice enhances germination and growth at low temperature (Takesawa et al., 2002). Therefore, the up-regulation in glutathione transerase activity in this study comes as no surprise.

\section{Photosynthesis and $\mathrm{CO}_{2}$ Fixation}

The down-regulation of terms related to photosynthesis and $\mathrm{CO}_{2}$ fixation are consistent with previous studies. Low temperature conditions are known to cause a reduction in maximum quantum yields for $\mathrm{CO}_{2}$ uptake, losses in the photochemical efficiency of photosystem II, and, with prolonged exposure to excessive light, a decreased rate of light saturated photosynthesis (Kratsch and Wise, 2000; Renaut et al., 2005). This phenomenon, photoinhibition, is associated with photodamage in plant cells and is usually used as a marker for cold tolerance (DemmigAdams and Adams Iii, 1992; Huner et al., 1993; Long et al., 1994).

Some authors describe photosynthesis as a sensor that is capable of regulating the imbalance between energy uptake and $\mathrm{CO}_{2}$ fixation (Huner et al., 1993). From this perspective, photoinhibition should be the consequence of a plant's capacity to adjust photosynthetically to the prevailing environmental conditions, rather than the result of damage or injury (Huner et al., 1993; Ensminger et al., 2006). However, photodamage still should not be discounted.

Proteomics analysis of Thellungiella halophila at low temperature demonstrated that half of the identified coldresponsive proteins were related to chloroplast physiology and function (Gao et al., 2009), suggesting at least partial regulation of cold stress tolerance through chloroplast metabolism. A comparable analysis of rice gave similar results (Hashimoto and Komatsu, 2007), further corroborating our data.

\section{Starch Metabolism}

Several studies have demonstrated a strong correlation between sugar concentration and cold stress tolerance (Tarkowski and Van den Ende, 2015). In fact, some key enzymes of this metabolism, specifically sucrose synthase [EC:2.4.1.13] and sucrose phosphate synthase [EC:2.4.1.14], can be regulated by low temperatures (Guy et al., 1992; Sasaki et al., 2001); however, this only applies to the first of these enzymes in this study (Table 1).

The role of starch and sucrose metabolism in this context has been reinforced in multiple mutant and transgenic plant studies. A mutant plant at the eskimo1 (esk1) locus, which exhibits higher levels of soluble sugars, was frost tolerant (Xin, 1998). On the other hand, an Arabidopsis mutant (sfru) that was deficient in tolerating freezing conditions after cold acclimation did not present cold-induced elevation of sucrose and glucose levels (Mckown et al., 1996). Meanwhile, Gilmour et al. (2000) studied the overexpression of the CBF3 TF in Arabidopsis, which confers cold tolerance, and found that total sugar levels were higher than those in control plants.

It could be argued that sucrose is a storage carbohydrate that can be easily catabolized when needed, making it useful either under stress or after leaving stress conditions (Pollock and Lloyd, 1987; Guy et al., 1992). Furthermore, the increase in these metabolite concentrations could be a way of maintaining carbon flux by compensating for $\mathrm{CO}_{2}$ assimilation and avoiding photoinhibition (Savitch et al., 2000). On the other hand, this upregulation could also be a way to cope with osmotic impairment generated by low temperature conditions (Anchordoguy et al., 1987; Palonen and Junttila, 1999) In such cases, plants can 
respond through an osmotic adjustment (Xiong and Zhu, 2002; Beck et al., 2007), in a process for which sugars could be a key component (Guy et al., 1992).

\section{Arginine and Proline Metabolism}

Proline is a major organic osmolyte that accumulates in a variety of plant species in response to environmental stresses, such as drought, salinity, and extreme temperatures (Hare and Cress, 1997; Ashraf and Foolad, 2007). In plants, L-proline is synthesized from L-glutamic acid via $\Delta^{1}$-pyrroline-5-carboxylate (P5C) through P5C synthetase (P5CS) and P5C reductase. Some authors have described a transcriptional regulation of the P5CS gene, the rate-limiting factor in proline biosynthesis, under osmotic stress (Yoshiba et al., 1995, 1997).

Yoshiba et al. (1995) did not find any induction of P5CS during cold stress of Arabidopsis thaliana. However, this contrasts with the up-regulation of this gene observed in this study, combined with an increase in proline content in different L. japonicus accessions after 7 days of cold stress (data not shown). Differences in P5CS gene transcriptional regulation are to be expected, due to differences in plant species, genotype, timing, and intensity of stress imposition.

Although the actual roles of proline in plant osmotolerance remain controversial, there is supporting evidence for a positive effect on enzyme and membrane integrity, osmotic adjustment, and free radical scavenging (Kishor et al., 2005). Still, some authors have argued that proline accumulation under stress is a product of, and not an adaptive response to stress (De Lacerda et al., 2003; Maiale et al., 2004). This uncertainty calls for further study of this feature, at least in the case of $L$. japonicus.

\section{Biosynthesis of Secondary Metabolites}

Most phenolic compounds in plants are derived from the phenylpropanoid pathway and have many different physiological roles. Increases in phenolic compound content under abiotic stress, particularly with respect to flavonoids, have been extensively described (Christie et al., 1994; Dixon and Paiva, 1995), making our results consistent with previous observations.

Among the enzymes involved in the phenylpropanoid biosynthesis pathway, phenylalanine ammonia-lyase [EC:4.3.1.24] is one of the most relevant (Rivero et al., 2001). This enzyme catalyzes the transformation of L-phenylalanine into trans-cinnamic acid, and has been shown to increase in activity in response to thermal stress (Leyva et al., 1995). However, we did not find any transcriptional regulation of this particular enzyme. Nevertheless, post-transcriptional regulation is possible.

Meanwhile, phenols are oxidized by peroxidases, which increase in activity in response to different types of stress (Jansen et al., 2001; Michalak, 2006). Most flavonoids outperform wellknown antioxidants, such as ascorbate, but different flavonoid oxidation processes could involve ROS scavenging as well (Hernández et al., 2009). Thus, peroxidase activity may play an essential role in these phenomena (Yamasaki et al., 1997).

Chalcone synthase [EC:2.3.1.74] and chalcone isomerase [EC:5.5.1.6] are codified by the $\mathrm{CHS}$ and $\mathrm{CHI}$ genes, respectively, and are key components of the flavonoid biosynthetic process; as such, their transcription is enhanced under cold stress (Wu et al., 2014), as was observed in this case.

\section{BR Biosynthesis and Steroid Hormone Biosynthesis}

BRs are steroidal plant hormones implicated in the promotion of plant growth and development, as well as in the stress response (Sasse, 1997). In fact, evidence suggests that BRs have a protecting effect at low temperatures, in that they activate coldstress related genes COR47 and COR78 (Müssig et al., 2002; Janeczko et al., 2007; Bajguz and Hayat, 2009). However, recent studies corroborate this one in suggesting that BR biosynthesis is down-regulated at low temperatures in certain plants (Gray and Heath, 2005; Hannah et al., 2005; Kaplan et al., 2007).

This response, seemingly the opposite of what would be beneficial, was reversed by Kim et al. (2010), who studied Arabidopsis with a BR-insensitive 1 (bri1) mutation. These plants suffered from defective BR signaling, which seemingly attributed to improve tolerance to cold stress.

The role of BR in the abiotic stress response is still not clear, but our results support a possible physiological relevance of this down-regulation.

\section{Organismal System Category}

Heat-shock proteins are molecular chaperones that cope with stress-induced denaturation of other proteins (Feder and Hofmann, 1999; Sun et al., 2002; Wang et al., 2004). Indeed, Sabehat et al. (1998) showed that an induced expression of two heat-shock proteins in Lycopersicon esculentum L. correlated with protection against some of the symptoms of chilling injuries.

Meanwhile, interleukin-1 receptor-associated kinase 4 [EC:2.7.11.1] (K04733) is a central part of the immune and inflammatory response in mammals (Janssens and Beyaert, 2003). The signaling domains of this kinase share significant homology with some of the plant proteins that mediate activation of mitogen-activated protein kinase (MAPK) pathways (Neill and Greene, 1998). These plant proteins have been related to pathogen attack response (Dardick and Ronald, 2006), as well as abiotic stress tolerance (Lehti-Shiu et al., 2009; Rodriguez et al., 2010). Particularly, Zou et al. (2006) showed an increased expression of a receptor-associated kinase of maize ( $\mathrm{mPti1}$ ) at low temperatures. In our study, the up-regulation of some of the aforementioned genes may reflect the induction of many mitogen-activated protein kinase signaling pathways as a consequence of cold stress imposition. However, the specific gene functions remain to be further studied.

\section{Up-Regulated TFs}

Genes that are members of the AP2/ERF family are plant-specific TFs that share a conserved DNA-binding domain and participate in the abiotic stress response via specific binding to DRE/CRT, a cis-acting element in the promoter's target genes (Mizoi et al., 2012). AP2/ERF can be divided into four different subfamilies, namely AP2, RAV, ERF, and DREB (Mizoi et al., 2012).

Some of the most described AP2/ERF TFs in the cold stress response network belong to the DREB1/CBF (A-1) subgroup (Yamaguchi-Shinozaki and Shinozaki, 2005; Nakashima and Yamaguchi-Shinozaki, 2006). Among these, CBF3, CBF1, and $\mathrm{CBF} 2$ are highly similar in amino acid sequence and are quickly and transiently induced by cold stress. Their expression is $\mathrm{ABA}$ independent and their products activate multiple stressinducible target genes (Mizoi et al., 2012). Interestingly, none 
of the AP2/ERF TFs that are up-regulated were DREB1/CBFs (Supplementary Table 3). Different authors have described characteristic expression profiles of these TFs within the first $8 \mathrm{~h}$ of stress (Novillo et al., 2004; Vogel et al., 2005), indicating that their expression should be studied at shorter intervals. Expression levels of some of these measured genes are analyzed in this way in the following section.

On the other hand, many studies demonstrate participation of the MYB TFs in different cold stress response pathways (Chinnusamy et al., 2007). Particularly, some are involved in the regulation of $\mathrm{CBF}$ TFs through the $\mathrm{ABA}$ independent signaling pathway (Chinnusamy et al., 2007). In fact, MYB15 negatively regulates the expression of the CBFs, as has been shown in a mutant and transgenic Arabidopsis with impaired MYB15 expression (Agarwal et al., 2006).

Some ABA-inducible MYB proteins may participate cooperatively in the ABA-dependent expression of different cold regulated genes as well (Shinozaki and Yamaguchishinozaki, 2000; Zhu et al., 2005; Dai et al., 2007). Because these MYB proteins are synthesized after endogenous levels of ABA accumulate, they do not play a signaling role until the later stages of the stress response. As a consequence, MYB TFs may participate in both the short and long term response to cold stress, and in an ABA-independent and/or dependent pathway.

Most of the other up-regulated TF families, such as WRKY, NAC, C2H2, and HSF, participate in different abiotic stresses responses (Chinnusamy et al., 2007; Chen et al., 2012; Scharf et al., 2012). Particularly, the NAC family may contribute to ABAdependent gene expression under various stresses, including cold (Tran et al., 2004; Yamaguchi-Shinozaki and Shinozaki, 2005; Nakashima et al., 2007, 2012).

Meanwhile, the calcium-calmodulin-CAMTA complex participates in the transcription regulation of different target genes. In this sense, cellular calcium(II) levels act as a secondary messenger that modulates diverse physiological processes that are important for stress adaptation. In particular, a rapid calcium influx is required for proper cold acclimation (Doherty et al., 2009).

In addition, a possible function of the calcium-calmodulinCAMTA complex was suggested in the DREB1/CBF signaling pathway (Thomashow, 2010). In fact, Doherty et al. (2009) proved that CBF1, CBF2, and ZAT12 were direct targets of CAMTA3 (Doherty et al., 2009). The evidence that CAMTA TFs and calcium signaling are upstream of the DREB1/CBF regulon make them relevant in the first few hours of stress imposition. However, this does not mean that calcium signaling does not play a role at longer timeframes. Thus, an induction in chr4.CM0307.390.r2.d gene expression may imply that calcium participates in the cold stress response in a DREB1/CBF independent pathway or downstream from their target genes, a hypothesis that is reinforced by our calmodulin gene expression data.

\section{The DREB1/CBF response}

The DREB1/CBF regulatory genes are an AP2/ERF TF subgroup that binds to the DRE/CRT regulatory element present in the promoters of target genes (Yamaguchi-Shinozaki and Shinozaki, 2005; Mizoi et al., 2012). These genes have been well characterized as key components of the ABA-independent cold stress response, mainly in Arabidopsis (Thomashow, 2010; Medina et al., 2011). In fact, a distinctive CBF pattern expression has been described for within the first $8 \mathrm{~h}$ (Novillo et al., 2004; Vogel et al., 2005). Remarkably, until now, no CBF expression studies have been performed for the Lotus genus.

The CBF1 and CBF3 results of this study are in agreement with those obtained by Novillo et al. (2004) and Cook et al. (2004), which showed maximum expression levels within the first $2 \mathrm{~h}$ of cold stress in Arabidopsis plants. Different authors have also described a similar expression pattern for $\mathrm{CBF} 2$, reaching a maximum only several hours later than CBF1 and CBF3 (Cook et al., 2004; Novillo et al., 2004, 2007). The observed differences in our study may be explained by the fact that most pertinent studies were completed using Arabidopsis, with only a few using legume species like Medicago spp. (Pennycooke et al., 2008; Zhang et al., 2011). In fact, there is no conclusive evidence for this expression pattern in L. japonicus. Interestingly, CBF2 has been described as a negative regulator of CBF1 and CBF3 (Novillo et al., 2004; Medina et al., 2011). As a consequence, the initial reduction observed in its expression level in L. japonicus might actually support the increase in CBF1 and CBF3.

Like CBF2, ZAT12 has been described as a negative regulator of DREB1/CBFs, though it is generally induced in this timeframe in Arabidopsis (Vogel et al., 2005; Zhai et al., 2010). However, there is no evidence of this TF behavior in legumes.

On the other hand, one TF that positively regulates DREB1/CBF TF expression is the MYC-like bHLH protein ICE1 (Yamaguchi-Shinozaki and Shinozaki, 2006). The relevance of this $\mathrm{TF}$ in cold stress response has been demonstrated by ice1 Arabidopsis mutants in which DREB1/CBFs expression was deregulated, leading to a reduced expression of many of their downstream cold-responsive genes (Chinnusamy et al., 2003; Lee et al., 2005). However, it was shown that a cold induced posttranslational modification of ICE1, rather than an increase in expression, allowed for the positive regulation of the DREB1/CBF regulon (Sharma et al., 2005). Further studies should be carried out to clarify the role of ICE1 in the cold stress response of $L$. japonicus.

Finally, the expression patterns of RD29A and COR47 are consistent with the fact that these are known cor genes that drive the cold stress response in plants several hours after stress conditions begin (Thomashow et al., 2001).

\section{CONCLUSION}

Our study provides novel insights into the molecular mechanisms of L. japonicus cold stress response.Overall, 1077 differentially expressed genes were obtained using RNA-Seq data, consisting of 713 that were up-regulated and 364 that were down-regulated. These genes were classified by their functional annotation through BLASTx, GO, and KEGG analysis.

Cold stress in L. japonicus modulates gene expression of lipid and cell wall metabolism, together with transcription regulation of genes related to flavonoid and phenylpropanoid biosynthesis. Genes related to xenobiotic degradation, heatshock proteins, and starch and proline metabolism were up-regulated. In contrast, photosynthesis and chloroplast 
related genes were down-regulated, demonstrating a dependency of cold acclimation on the photosynthetic process.

Different types of TFs were up-regulated as well, with the MYB, AP2/ERF, and NAC families being the most numerous. Interestingly, two putative novel TFs were identified that participate in cold response. Finally, the expression profiles of some DREB1/CBFs and cor genes were described for the first $8 \mathrm{~h}$ of stress. To our knowledge, this is the first report of DREB1/CBF expression under low temperature conditions in this genus.

Our results constitute the first transcriptome profiling of the model legume L. japonicus under cold stress. Data obtained allowed for the identification of possible target metabolisms, information that could be used to improve stress tolerance in L. japonicus, not to mention other members of the genus, in the future.

\section{AUTHOR CONTRIBUTIONS}

PC, SM, OR, and FE conceived and designed the experiments. PC performed the experiments. PC and FE analyzed the data. OR contributed reagents, materials, and analytical tools. PC, SM, OR, and FE wrote the paper. All authors read and approved the final manuscript.

\section{REFERENCES}

Agarwal, M., Hao, Y., Kapoor, A., Dong, C.-H., Fujii, H., Zheng, X., et al. (2006). A R2R3 type MYB transcription factor is involved in the cold regulation of CBF genes and in acquired freezing tolerance. J. Biol. Chem. 281, 37636-37645. doi: 10.1074/jbc.M605895200

Al-Shahrour, F., Díaz-Uriarte, R., and Dopazo, J. (2004). FatiGO: a web tool for finding significant associations of Gene Ontology terms with groups of genes. Bioinformatics 20, 578-580. doi: 10.1093/bioinformatics/btg455

Anchordoguy, T. J., Rudolph, A. S., Carpenter, J. F., and Crowe, J. H. (1987). Modes of interaction of cryoprotectants with membrane phospholipids during freezing. Cryobiology 24, 324-331.

Anderson, J. V., and Davis, D. G. (2004). Abiotic stress alters transcript profiles and activity of glutathione S-transferase, glutathione peroxidase, and glutathione reductase in Euphorbia esula. Physiol. Plant. 120, 421-433. doi: 10.1111/j.00319317.2004.00249.x

Andrews, S. (2010). FastQC: A Quality Control Tool for High Throughput Sequence Data. Available online at: http://www.bioinformatics.babraham.ac.uk/projects/ fastqc/

Apel, K., and Hirt, H. (2004). Reactive oxygen species: metabolism, oxidative stress, and signal transduction. Annu. Rev. Plant Biol. 55, 373-399. doi: 10.1146/annurev.arplant.55.031903.141701

Ashraf, M., and Foolad, M. R. (2007). Roles of glycine betaine and proline in improving plant abiotic stress resistance. Environ. Exp. Bot. 59, 206-216. doi: 10.1016/j.envexpbot.2005.12.006

Babuin, M. F., Campestre, M. P., Rocco, R., Bordenave, C. D., Escaray, F. J., Antonelli, C., et al. (2014). Response to long-term NaHCO3-derived alkalinity in model Lotus japonicus Ecotypes Gifu B-129 and Miyakojima MG-20: transcriptomic profiling and physiological characterization. PLoS ONE 9:e97106. doi: 10.1371/journal.pone.0097106

Bajguz, A., and Hayat, S. (2009). Effects of brassinosteroids on the plant responses to environmental stresses. Plant Physiol. Biochem. 47, 1-8. doi: 10.1016/j.plaphy.2008.10.002

Beck, E. H., Fettig, S., Knake, C., Hartig, K., and Bhattarai, T. (2007). Specific and unspecific responses of plants to cold and drought stress. J. Biosci. 32, 501-510. doi: $10.1007 /$ s12038-007-0049-5

\section{FUNDING}

CONICET (PIP 0980). ANPCYT (PICT 1612)-PhD Oscar Adolfo

Ruiz.

\section{AUTHOR INFORMATION}

PC is a CONICET fellow. SM, OR, and FE are career research members of CONICET.

\section{ACKNOWLEDGMENTS}

This work was supported by the following grants: PICT of Agencia Nacional de Promoción Científica y Tecnológica (ANPCYT, Argentina), Consejo Nacional de Investigaciones Científicas y Técnicas (CONICET, Argentina), and Universidad de San Martín (UNSAM). 3PA. We would also like to thank Daniel Davis for English language and general editing of the manuscript.

\section{SUPPLEMENTARY MATERIAL}

The Supplementary Material for this article can be found online at: http://journal.frontiersin.org/article/10.3389/fpls.2016. 00374

Blumenthal, M. J., and McGraw, R. L. (1999). "Lotus adaptation, use, and management," in Trefoil: The Science and Technology of Lotus (Madison, WI: Crop Science Society of America and American Society of Agronomy), 97-119.

Bullard, M. J., and Crawford, T. J. (1995). Productivity of Lotus corniculatus L. (bird's-foot trefoil) in the UK when grown under low-input conditions as spaced plants, monoculture swards or mixed swards. Grass Forage Sci. 50, 439-446.

Chalker-Scott, L. (1999). Environmental significance of anthocyanins in plant stress responses. Photochem. Photobiol. 70, 1. doi: 10.1562/00318655(1999) $070<0001$ :ESOAIP > 2.3.CO;2

Chen, L., Song, Y., Li, S., Zhang, L., Zou, C., and Yu, D. (2012). The role of WRKY transcription factors in plant abiotic stresses. Biochim. Biophys. Acta 1819, 120-128. doi: 10.1016/j.bbagrm.2011.09.002

Chinnusamy, V., Ohta, M., Kanrar, S., Lee, B., Hong, X., Agarwal, M., et al. (2003). ICE1?: a regulator of cold-induced transcriptome and freezing tolerance in Arabidopsis. Genes Dev. 17, 1043-1054. doi: 10.1101/gad.1077503.CRT

Chinnusamy, V., Zhu, J., and Zhu, J.-K. (2007). Cold stress regulation of gene expression in plants. Trends Plant Sci. 12, 444-451. doi: 10.1016/j.tplants.2007.07.002

Christie, P. J., Alfenito, M. R., and Walbot, V. (1994). Impact of low-temperature stress on general phenylpropanoid and anthocyanin pathways?: enhancement of transcript abundance and anthocyanin pigmentation in maize seedlings. Planta 194, 541-549.

Conesa, A., Götz, S., García-Gómez, J. M., Terol, J., Talón, M., and Robles, M. (2005). Blast2GO: a universal tool for annotation, visualization and analysis in functional genomics research. Bioinformatics 21, 3674-3676. doi: 10.1093/bioinformatics/bti610

Cook, D., Fowler, S., Fiehn, O., and Thomashow, M. F. (2004). A prominent role for the $\mathrm{CBF}$ cold response pathway in configuring the low-temperature metabolome of Arabidopsis. Proc. Natl. Acad. Sci. U.S.A. 101, 15243-15248. doi: 10.1073/pnas.0406069101

Dai, X., Xu, Y., Ma, Q., Xu, W., Wang, T., Xue, Y., et al. (2007). Overexpression of an R1R2R3 MYB gene, OsMYB3R-2, increases tolerance to freezing, drought, and salt stress in transgenic Arabidopsis. Plant Physiol. 143, 1739-1751. doi: $10.1104 /$ pp.106.094532 
Dardick, C., and Ronald, P. (2006). Plant and animal pathogen recognition receptors signal through non-RD kinases. PLoS Pathog. 2:e2. doi: 10.1371/journal.ppat.0020002

De Lacerda, C. J. C., Oliva, M., Ruiz, H., and Prisco, J. (2003). Solute accumulation and distribution during shoot and leaf development in two sorghum genotypes under salt stress. Environ. Exp. Bot. 49, 107-120. doi: 10.1016/S00988472(02)00064-3

Demmig-Adams, B., and Adams Iii, W. W. (1992). Photoprotection and other responses of plants to high light stress. Annu. Rev. Plant Biol. 43, 599-626.

Deyholos, M. K. (2010). Making the most of drought and salinity transcriptomics. Plant Cell Environ. 33, 648-654. doi: 10.1111/j.1365-3040.2009.02092.x

Díaz, P., Betti, M., Sánchez, D. H., Udvardi, M. K., Monza, J., and Márquez, A. J. (2010). Deficiency in plastidic glutamine synthetase alters proline metabolism and transcriptomic response in Lotus japonicus under drought stress. New Phytol. 188, 1001-1013. doi: 10.1111/j.1469-8137.2010.03440.x

Dinari, A., Niazi, A., Afsharifar, A. R., and Ramezani, A. (2013). Identification of upregulated genes under cold stress in cold-tolerant chickpea using the cDNAAFLP approach. PLoS ONE 8:e52757. doi: 10.1371/journal.pone.0052757

Di Rienzo, J. A., Casanoves, F., Balzarini, M. G., Gonzalez, L., Tablada, M., and Robledo, C. W. (2008). InfoStat, versión 2008. Argentina: Grupo InfoStat, FCA, Universidad Nacional de Córdoba.

Dixon, D. P., Cummins, I., Cole, J. D., and Robert, E. (1998). Glutathione-mediated detoxification systems in plants David. Curr. Opin. Plant Biol. 1, 258-266.

Dixon, R. A., and Paiva, N. L. (1995). Stress-lnduced phenylpropanoid metabolism. Plant Cell 7, 1085-1097.

Doherty, C. J., Van Buskirk, H. A., Myers, S. J., and Thomashow, M. F. (2009). Roles for Arabidopsis CAMTA transcription factors in coldregulated gene expression and freezing tolerance. Plant Cell 21, 972-984. doi: $10.1105 /$ tpc. 108.063958

Domon, J.-M., Baldwin, L., Acket, S., Caudeville, E., Arnoult, S., Zub, H., et al. (2013). Cell wall compositional modifications of Miscanthus ecotypes in response to cold acclimation. Phytochemistry 85, 51-61. doi: 10.1016/j.phytochem.2012.09.001

Donà, M., Confalonieri, M., Minio, A., Biggiogera, M., Buttafava, A., Raimondi, E., et al. (2013). RNA-Seq analysis discloses early senescence and nucleolar dysfunction triggered by Tdp1 $\alpha$ depletion in Medicago truncatula. J. Exp. Bot. 64, 1941-1951. doi: 10.1093/jxb/ert063

Dong, J., Jiang, Y., Chen, R., Xu, Z., and Gao, X. (2013). Isolation of a novel xyloglucan endotransglucosylase (OsXET9) gene from rice and analysis of the response of this gene to abiotic stresses. Afr. J. Biotechnol. 10, 17424-17434. doi: 10.5897/AJB11.1242

Ensminger, I., Busch, F., and Huner, N. P. A. (2006). Photostasis and cold acclimation: sensing low temperature through photosynthesis. Physiol. Plant. 126, 28-44. doi: 10.1111/j.1399-3054.2006.00627.x

Escaray, F. J., Menendez, A. B., Gárriz, A., Pieckenstain, F. L., Estrella, M. J., Castagno, L. N., et al. (2012). Ecological and agronomic importance of the plant genus Lotus. Its application in grassland sustainability and the amelioration of constrained and contaminated soils. Plant Sci. 182, 121-133. doi: 10.1016/j.plantsci.2011.03.016

Escaray, F. J., Passeri, V., Babuin, F. M., Marco, F., Carrasco, P., Damiani, F., et al. (2014). Lotus tenuis $\mathrm{x}$ L. corniculatus interspecific hybridization as a means to breed bloat-safe pastures and gain insight into the genetic control of proanthocyanidin biosynthesis in legumes. BMC Plant Biol. 14:40. doi: 10.1186/1471-2229-14-40

Feder, M. E., and Hofmann, G. E. (1999). Heat-shock proteins, molecular chaperones, and the stress response?: evolutionary and ecological physiology. Annu. Rev. Physiol. 61, 243-282.

Fukai, E., Soyano, T., Umehara, Y., Nakayama, S., Hirakawa, H., Tabata, S., et al. (2012). Establishment of a Lotus japonicus gene tagging population using the exon-targeting endogenous retrotransposon LORE1. Plant J. 69, 720-730. doi: 10.1111/j.1365-313X.2011.04826.x

Gall, H., Philippe, F., Domon, J.-M., Gillet, F., Pelloux, J., and Rayon, C. (2015). Cell wall metabolism in response to abiotic stress. Plants 4, 112-166. doi: 10.3390/plants4010112

Gao, F., Zhou, Y., Zhu, W., Li, X., Fan, L., and Zhang, G. (2009). Proteomic analysis of cold stress-responsive proteins in Thellungiella rosette leaves. Planta 230, 1033-1046. doi: 10.1007/s00425-009-1003-6

Gilmour, S. J., Sebolt, A. M., Salazar, M. P., Everard, J. D., and Thomashow, M. F. (2000). Overexpression of the Arabidopsis CBF3 transcriptional activator mimics multiple biochemical changes associated with cold acclimation. Plant Physiol. 124, 1854-1865. doi: 10.1104/pp.124.4.1854

Gray, G. R., and Heath, D. (2005). A global reorganization of the metabolome in Arabidopsis during cold acclimation is revealed by metabolic fingerprinting. Physiol. Plant. 124, 236-248. doi: 10.1111/j.1399-3054.2005.00507.x

Guy, C. L., Huber, J. L. A., and Huber, S. C. (1992). Sucrose phosphate synthase and sucrose accumulation at low temperature'. Plant Physiol. 100, 502-508.

Halling, M. A., Topp, C. F. E., and Doyle, C. J. (2004). Aspects of the productivity of forage legumes in Northern Europe. Gras 59, 331-344. doi: 10.1111/j.13652494.2004.00435.x

Halusková, L., Valentovicová, K., Huttová, J., Mistrík, I., and Tamás, L. (2009). Effect of abiotic stresses on glutathione peroxidase and glutathione Stransferase activity in barley root tips. Plant Physiol. Biochem. 47, 1069-1074. doi: 10.1016/j.plaphy.2009.08.003

Handberg, K., and Stougaard, J. (1992). Lotus japonicus, an autogamous, diploid legume species for classical and molecular genetics. Plant J. 2, 487-496.

Hannah, M., a, Heyer, A. G., and Hincha, D. K. (2005). A global survey of gene regulation during cold acclimation in Arabidopsis thaliana. PLoS Genetics 1:e26. doi: 10.1371/journal.pgen.0010026

Hare, P. D., and Cress, W. A. (1997). Metabolic implications of stress-induced proline accumulation in plants. Plant Growth Regul. 21, 79-102.

Hashimoto, M., and Komatsu, S. (2007). Proteomic analysis of rice seedlings during cold stress. Proteomics 7, 1293-1302. doi: 10.1002/pmic.200600921

Hernández, I., Alegre, L., Van Breusegem, F., and Munné-Bosch, S. (2009). How relevant are flavonoids as antioxidants in plants? Trends Plant Sci. 14, 125-132. doi: 10.1016/j.tplants.2008.12.003

Hietala, T., Mozes, N., Genet, M., Rosenqvist, H., and Laakso, S. (1997). Surface lipids and their distribution on willow (Salix) leaves: a combined chemical, morphological and physicochemical study. Colloids Surfaces B 8, 205-215.

Hoagland, D. R., and Arnon, D. I. (1950). The Water-Culture Method for Growing Plants Without Soil, 2nd Edn., Vol. 347. Circular. Berkeley: California Agricultural Experiment Station, 32.

Huner, N. P. A., Oquist, G., Hurry, V. M., Krol, M., Falk, S., and Griffith, M. (1993). Photosynthesis, photoinhibition and low temperature acclimation in cold tolerant plants. Photosyn. Res. 37, 19-39.

Iba, K. (2002). Acclimative response to temperature stress in higher plants: approaches of gene engineering for temperature tolerance. Annu. Rev. Plant Biol. 53, 225-245. doi: 10.1146/annurev.arplant.53.100201.160729

Janeczko, A., Gullner, G., Skoczowski, A., Dubert, F., and Barna, B. (2007). Effects of brassinosteroid infiltration prior to cold treatment on ion leakage and pigment contents in rape leaves. Biol. Plantarum 51, 355-358. doi: 10.1007/s10535-007-0072-2

Jansen, M. A. K., Van Den Noort, R. E., Tan, M. Y. A., Prinsen, E., Lagrimini, L. M., and Thorneley, R. N. F. (2001). Phenol-oxidizing peroxidases contribute to the protection of plants from ultraviolet radiation stress. Plant Physiol. 126, 1012-1023. doi: 10.1104/pp.126.3.1012

Janssens, S., and Beyaert, R. (2003). Functional diversity and regulation of different Interleukin-1 receptor- associated kinase (IRAK) family members. Mol. Cell. 11, 293-302. doi: 10.1016/S1097-2765(03)00053-4

Jin, J., Zhang, H., Kong, L., Gao, G., and Luo, J. (2014). PlantTFDB 3.0: a portal for the functional and evolutionary study of plant transcription factors. Nucleic Acids Res. 42, D1182-D1187. doi: 10.1093/nar/gkt1016

Kakumanu, A., Ambavaram, M. M. R., Klumas, C., Krishnan, A., Batlang, U., Myers, E., et al. (2012). Effects of drought on gene expression in maize reproductive and leaf meristem tissue revealed by RNA-Seq. Plant Physiol. 160, 846-867. doi: 10.1104/pp.112.200444

Kanehisa, M., Goto, S., Furumichi, M., Tanabe, M., and Hirakawa, M. (2010). KEGG for representation and analysis of molecular networks involving diseases and drugs. Nucleic Acids Res. 38, D355-D360. doi: 10.1093/nar/gkp896

Kaplan, F., Kopka, J., Sung, D. Y., Zhao, W., Popp, M., Porat, R., et al. (2007). Transcript and metabolite profiling during cold acclimation of Arabidopsis reveals an intricate relationship of cold-regulated gene expression with modifications in metabolite content. Plant J. 50, 967-981. doi: 10.1111/j.1365313X.2007.03100.x

Kim, S. Y., Kim, B. H., Lim, C. J., Lim, C. O., and Nam, K. H. (2010). Constitutive activation of stress-inducible genes in a brassinosteroid-insensitive 1 (bri1) mutant results in higher tolerance to cold. Physiol. Plant. 138, 191-204. doi: 10.1111/j.1399-3054.2009.01304.x 
Kishor, P. B. K., Sangam, S., Amrutha, R. N., Laxmi, P. S., Naidu, K. R., Rao, K. R. S. S., et al. (2005). Regulation of proline biosynthesis, degradation, uptake and transport in higher plants?: Its implications in plant growth and abiotic stress tolerance. Curr. Sci. 88, 424-438.

Kocsy, G., Galiba, G., and Brunold, C. (2001). Role of glutathione in adaptation and signalling during chilling and cold acclimation in plants. Physiol. Plant. 113, 158-164. doi: 10.1034/j.1399-3054.2001.1130202.x

Kratsch, H. A., and Wise, R. R. (2000). The ultrastructure of chilling stress. Plant Cell Environ. 23, 337-350. doi: 10.1046/j.1365-3040.2000.00560.x

Lee, B., Henderson, D. A., and Zhu, J. (2005). The Arabidopsis cold-responsive transcriptome and its regulation by ICE1. Plant Cell 17, 3155-3175. doi: 10.1105/tpc.105.035568.1

Lehti-Shiu, M. D., Zou, C., Hanada, K., and Shiu, S.-H. (2009). Evolutionary history and stress regulation of plant receptor-like kinase/pelle genes. Plant Physiol. 150, 12-26. doi: 10.1104/pp.108.134353

Leyva, A., Jarillo, J. A., Salinas, J., and Martinez-Zapater, J. M. (1995). Low temperature induces the accumulation of phenylalanine ammonia-lyase and chalcone synthase mRNAs of Arabidopsis thaliana in a light-dependent manner. Plant Physiol. 108, 39-46.

Li, J., Dai, X., Liu, T., and Zhao, P. X. (2012). LegumeIP: an integrative database for comparative genomics and transcriptomics of model legumes. Nucleic Acids Res. 40, D1221-D1229. doi: 10.1093/nar/gkr939

Li, X., Lei, M., Yan, Z., Wang, Q., Chen, A., Sun, J., et al. (2014). The REL3mediated TAS3 ta-siRNA pathway integrates auxin and ethylene signaling to regulate nodulation in Lotus japonicus. New Phytol. 201, 531-544. doi: $10.1111 / \mathrm{nph} .12550$

Long, S. P., Humphries, S., and Falkowski, P. G. (1994). Photoinhibition of photosynthesis in nature. Annu. Rev. Plant Biol. 45, 633-662.

López, M., Herrera-Cervera, J., a, Iribarne, C., Tejera, N., a, and Lluch, C. (2008). Growth and nitrogen fixation in Lotus japonicus and Medicago truncatula under $\mathrm{NaCl}$ stress: nodule carbon metabolism. J. Plant Physiol. 165, 641-650. doi: 10.1016/j.jplph.2007.05.009

Lucau-Danila, A., Toitot, C., Goulas, E., Blervacq, a, S., and Hot, D., Bahrman, N., et al., (2012). Transcriptome analysis in pea allows to distinguish chilling and acclimation mechanisms. Plant Physiol. Biochem. 58, 236-244. doi: 10.1016/j.plaphy.2012.07.012

Maiale, S., Sánchez, D. H., Guirado, A., Vidal, A., and Ruiz, O. A. (2004). Spermine accumulation under salt stress. J. Plant Physiol. 161, 35-42. doi: 10.1078/01761617-01167

Maruyama, K., Takeda, M., Kidokoro, S., Yamada, K., Sakuma, Y., Urano, K., et al. (2009). Metabolic pathways involved in cold acclimation identified by integrated analysis of metabolites and transcripts regulated by DREB1A and DREB2A. Plant Physiol. 150, 1972-1980. doi: 10.1104/pp.109.135327

Mckown, R., Kuroki, G., and Warren, G. (1996). Cold responses of Arabidopsis mutants impaired in freezing tolerance. J. Exp. Bot. 47, 1919-1925.

Medina, J., Catal,á, R., and Salinas, J. (2011). The CBFs: three arabidopsis transcription factors to cold acclimate. Plant Sci. 180, 3-11. doi: 10.1016/j.plantsci.2010.06.019

Michalak, A. (2006). Phenolic compounds and their antioxidant activity in plants growing under heavy metal stress. Polish J. Environ. Stud. 15, 523-530.

Mizoi, J., Shinozaki, K., and Yamaguchi-Shinozaki, K. (2012). AP2/ERF family transcription factors in plant abiotic stress responses. Biochim. Biophys. Acta 1819, 86-96. doi: 10.1016/j.bbagrm.2011.08.004

Moriya, Y., Itoh, M., Okuda, S., Yoshizawa, A. C., and Kanehisa, M. (2007). KAAS: an automatic genome annotation and pathway reconstruction server. Nucleic Acids Res. 35, W182-W185. doi: 10.1093/nar/gkm321

Müssig, C., Fischer, S., and Altmann, T. (2002). Brassinosteroid-regulated gene expression. Plant Physiol. 129, 1241-1251. doi: 10.1104/pp. 011003

Nakashima, K., Takasaki, H., Mizoi, J., Shinozaki, K., and Yamaguchi-Shinozaki, K. (2012). NAC transcription factors in plant abiotic stress responses. Biochim. Biophys. Acta 1819, 97-103. doi: 10.1016/j.bbagrm.2011.10.005

Nakashima, K., Tran, L.-S. P., Van Nguyen, D., Fujita, M., Maruyama, K., Todaka, D., et al. (2007). Functional analysis of a NAC-type transcription factor OsNAC6 involved in abiotic and biotic stress-responsive gene expression in rice. Plant J. 51, 617-630. doi: 10.1111/j.1365-313X.2007.03168.x

Nakashima, K., and Yamaguchi-Shinozaki, K. (2006). Regulons involved in osmotic stress-responsive and cold stress-responsive gene expression in plants. Physiol. Plant. 126, 62-71. doi: 10.1111/j.1399-3054.2005.00592.x
Neill, L. A. J. O., and Greene, C. (1998). Signal transduction pathways activated by the IL-1 receptor family?: ancient signaling machinery in mammals, insects, and plants. J. Leukoc. Biol. 63, 650-657.

Novillo, F., Alonso, J. M., Ecker, J. R., and Salinas, J. (2004). CBF2/DREB1C is a negative regulator of CBF1/DREB1B and CBF3/DREB1A expression and plays a central role in stress tolerance in Arabidopsis. Proc. Natl. Acad. Sci. U.S.A. 101, 3985-3990. doi: 10.1073/pnas.0303029101

Novillo, F., Medina, J., and Salinas, J. (2007). Arabidopsis CBF1 and CBF3 have a different function than CBF2 in cold acclimation and define different gene classes in the CBF regulon. Proc. Natl. Acad. Sci. U.S.A. 104, 21002-21007. doi: 10.1073/pnas.0705639105

Palonen, P., and Junttila, O. (1999). Cold hardening of raspberry plants in vitro is enhanced by increasing sucrose in the culture medium. Physiol. Plant. 106, 386-392.

Pang, T., Ye, C.-Y., Xia, X., and Yin, W. (2013). De novo sequencing and transcriptome analysis of the desert shrub, Ammopiptanthus mongolicus, during cold acclimation using Illumina/Solexa. BMC Genomics 14:488. doi: 10.1186/1471-2164-14-488

Pennycooke, J. C., Cheng, H., and Stockinger, E. J. (2008). Comparative genomic sequence and expression analyses of Medicago truncatula and alfalfa subspecies falcata COLD-ACCLIMATION-SPECIFIC genes. Plant Physiol. 146, 1242-1254. doi: 10.1104/pp.107.108779

Pollock, C. J., and Lloyd, E. J. (1987). The effect of low temperature upon starch, sucrose and fructan synthesis in leaves. Ann. Bot. 60, 231-235.

Renaut, J., Hoffmann, L., and Hausman, J.-F. (2005). Biochemical and physiological mechanisms related to cold acclimation and enhanced freezing tolerance in poplar plantlets. Physiol. Plant. 125, 82-94. doi: 10.1111/j.13993054.2005.00554.x

Rivero, R. M., Ruiz, J. M., García, P. C., López-Lefebre, L. R., Sánchez, E., and Romero, L. (2001). Resistance to cold and heat stress?: accumulation of phenolic compounds in tomato and watermelon plants. Plant Sci. 160, 315-321. doi: 10.1016/S0168-9452(00)00395-2

Rodriguez, M. C. S., Petersen, M., and Mundy, J. (2010). Mitogen-activated protein kinase signaling in plants. Annu. Rev. Plant Biol. 61, 621-649. doi: 10.1146/annurev-arplant-042809-112252

Sabehat, A., Lurie, S., and Weiss, D. (1998). Expression of small heat-shock proteins at low temperatures a possible role in protecting against chilling injuries. Plant Physiol. 117, 651-658. doi: 10.1104/pp.117.2.651

Sainz, M., Díaz, P., Monza, J., and Borsani, O. (2010). Heat stress results in loss of chloroplast $\mathrm{Cu} / \mathrm{Zn}$ superoxide dismutase and increased damage to Photosystem II in combined drought-heat stressed Lotus japonicus. Physiol. Plant. 140, 46-56. doi: 10.1111/j.1399-3054.2010.01383.x

Sasaki, H., Ichimura, K., Imada, S., and Yamaki, S. (2001). Sucrose synthase and sucrose phosphate synthase, but not acid invertase, are regulated by cold acclimation and deacclimation in cabbage seedlings. J. Plant Physiol. 158, 847-852. doi: 10.1078/0176-1617-00391

Sasse, J. (1997). Recent progress in brassinosteroid research. Physiol. Plant. 100, 696-701.

Sato, S., and Andersen, S. U. (2014). "Genome sequencing," in The Lotus japonicus Genome, Compendium of Plant Genomes, eds S. Tabata and J. Stougaard (Berlin: Springer), 35-40. doi: 10.1007/978-3-662-44270-8_4

Sato, S., Nakamura, Y., Kaneko, T., Asamizu, E., Kato, T., Nakao, M., et al. (2008). Genome structure of the legume, Lotus japonicus. DNA Res. 15, 227-239. doi: 10.1093/dnares/dsn008

Savitch, L. V., Harney, T., and Huner, N. P. A. (2000). Sucrose metabolism in spring and winter wheat in response to high irradiance, cold stress and cold acclimation. Physiol. Plant. 108, 270-278. doi: 10.1034/j.13993054.2000.108003270.x

Scharf, K.-D., Berberich, T., Ebersberger, I., and Nover, L. (2012). The plant heat stress transcription factor (Hsf) family: structure, function and evolution. Biochim. Biophys. Acta 1819, 104-119. doi: 10.1016/j.bbagrm.2011.10.002

Sharma, P., Sharma, N., and Deswal, R. (2005). The molecular biology of the low-temperature response in plants. Bioessays? 27, 1048-1059. doi: 10.1002/bies.20307

Shepherd, T., and Wynne Griffiths, D. (2006). The effects of stress on plant cuticular waxes. New Phytol. 171, 469-499. doi: 10.1111/j.14698137.2006.01826.x

Shinozaki, K., and Yamaguchi-shinozaki, K. (2000). Molecular responses to dehydration and low temperature?: differences and cross-talk between 
two stress signaling pathways. Curr. Opin. Plant Biol. 3, 217-223. doi: 10.1016/S1369-5266(00)00067-4

Shinozaki, K., Yamaguchi-Shinozaki, K., and Seki, M. (2003). Regulatory network of gene expression in the drought and cold stress responses. Curr. Opin. Plant Biol. 6, 410-417. doi: 10.1016/S1369-5266(03)00092-X

Smolenska, G., and Kuiper, P. J. C. (1977). Effect of low temperature upon lipid and fatty acid composition of roots and leaves of winter rape plants. Physiol. Plant. 41, 29-35.

Steponkus, P. L. (1984). Role of the plasma membrane in freezing injury and cold acclimation. Annu. Rev. Plant Physiol. 35, 543-584.

Sun, W., Montagu, M., Van, and Verbruggen, N. (2002). Small heat shock proteins and stress tolerance in plants. Biochem. Biophys. Acta 1577, 1-9. doi: 10.1016/s0167-4781(02)00417-7

Takesawa, T., Ito, M., Kanzaki, H., Kameya, N., and Nakamura, I. (2002). Over-expression of $\zeta$ glutathione S-transferase in transgenic rice enhances germination and growth at low temperature. Mol. Breeding 9, 93-101. doi: 10.1023/A:1026718308155

Tarkowski, Ł. P., and Van den Ende, W. (2015). Cold tolerance triggered by soluble sugars: a multifaceted countermeasure. Front. Plant Sci. 6:203. doi: $10.3389 /$ fpls.2015.00203

Team, R. C. (2014). R: A Language and Environment for Statistical Computing. Vienna: R Foundation for Statistical Computing.

Thakur, P., Kumar, S., Malik, J., a., Berger, J. D., and Nayyar, H. (2010). Cold stress effects on reproductive development in grain crops: an overview. Environ. Exp. Bot. 67, 429-443. doi: 10.1016/j.envexpbot.2009.09.004

Thomashow, M. F. (1999). Plant cold acclimation: freezing tolerance genes and regulatory mechanisms. Annu. Rev. Plant Biol. 50, 571-599.

Thomashow, M. F. (2010). Molecular basis of plant cold acclimation: insights gained from studying the CBF cold response pathway. Plant Physiol. 154, 571-577. doi: 10.1104/pp.110.161794

Thomashow, M. F., Gilmour, S. J., Stockinger, E. J., Jaglo-ottosen, K. R., and Zarka, D. G. (2001). Role of the Arabidopsis CBF transcriptional activators in cold acclimation. Physiol. Plant. 112, 171-175. doi: 10.1034/j.13993054.2001.1120204.x

Tran, L. P., Nakashima, K., Sakuma, Y., Simpson, S. D., Fujita, Y., Maruyama, K., et al. (2004). Isolation and functional analysis of Arabidopsis stressinducible NAC transcription factors that bind to a drought-responsive ciselement in the early responsive to dehydration stress 1 promoter. Plant Cell 16, 2481-2498. doi: 10.1105/tpc.104.022699

Trapnell, C., Roberts, A., Goff, L., Pertea, G., Kim, D., Kelley, D. R., et al. (2012). Differential gene and transcript expression analysis of RNASeq experiments with TopHat and Cufflinks. Nat. Protoc. 7, 562-578. doi: 10.1038/nprot.2012.016

Untergasser, A., Cutcutache, I., Koressaar, T., Ye, J., Faircloth, B. C., Remm, M., et al. (2012). Primer3-new capabilities and interfaces. Nucleic Acids Res. 40, e115-e115. doi: 10.1093/nar/gks596

Urbañski, D. F., Małolepszy, A., Stougaard, J., and Andersen, S. U. (2012). Genome-wide LORE1 retrotransposon mutagenesis and high-throughput insertion detection in Lotus japonicus. Plant J. 69, 731-741. doi: 10.1111/j.1365313X.2011.04827.x

Vogel, J. T., Zarka, D. G., Van Buskirk, H., a, Fowler, S. G., and Thomashow, M. F. (2005). Roles of the CBF2 and ZAT12 transcription factors in configuring the low temperature transcriptome of Arabidopsis. Plant J. 41, 195-211. doi: 10.1111/j.1365-313X.2004.02288.x

Wang, W., Vinocur, B., Shoseyov, O., and Altman, A. (2004). Role of plant heatshock proteins and molecular chaperones in the abiotic stress response. Trends Plant Sci. 9, 244-252. doi: 10.1016/j.tplants.2004.03.006

Wang, X.-C., Zhao, Q.-Y., Ma, C.-L., Zhang, Z.-H., Cao, H.-L., Kong, Y.-M., et al. (2013). Global transcriptome profiles of Camellia sinensis during cold acclimation. BMC Genomics 14:415. doi: 10.1186/1471-2164-14-415

Welti, R., Li, W., Li, M., Sang, Y., Biesiada, H., Zhou, H.-E., et al. (2002). Profiling membrane lipids in plant stress responses. Role of phospholipase D alpha in freezing-induced lipid changes in Arabidopsis. J. Biol. Chem. 277, 31994-32002. doi: 10.1074/jbc.M205375200

Wheeler, T. R., Craufurd, P. Q., Ellis, R. H., Porter, J. R., and Prasad, P. V. V. (2000). Temperature variability and the yield of annual crops. Agricult. Ecosyst. Environ. 82, 159-167. doi: 10.1016/S0167-8809(00)00224-3
Willemot, C., Hope, H. J., Williams, R. J., and Michaud, R. (1977). Changes in fatty acid composition of winter wheat during frost hardening. Cryobiology 14, 87-93.

Wu, Y., Wei, W., Pang, X., Wang, X., Zhang, H., Dong, B., et al. (2014). Comparative transcriptome profiling of a desert evergreen shrub, Ammopiptanthus mongolicus, in response to drought and cold stresses. BMC Genomics 15:671. doi: 10.1186/1471-2164-15-671

Xin, Z. (1998). eskimol mutants of Arabidopsis are constitutively freezingtolerant. Proc. Natl. Acad. Sci. 95, 7799-7804.

Xiong, L., and Zhu, J. (2002). Molecular and genetic aspects of plant responses to osmotic stress. Plant Cell Environ. 25, 131-139. doi: 10.1046/j.13653040.2002.00782.x

Yamaguchi-Shinozaki, K., and Shinozaki, K. (2005). Organization of cis-acting regulatory elements in osmotic-and cold-stress-responsive promoters. Trends Plant Sci. 10, 88-94. doi: 10.1016/j.tplants.2004.12.012

Yamaguchi-Shinozaki, K., and Shinozaki, K. (2006). Transcriptional regulatory networks in cellular responses and tolerance to dehydration and cold stresses. Annu. Rev. Plant Biol. 57, 781-803. doi: 10.1146/annurev.arplant.57.032905.105444

Yamasaki, H., Sakihama, Y., and Ikehara, N. (1997). Flavonoid-peroxidase reaction as a detoxification mechanism of plant cells against H2O2. Plant Physiol. 115, 1405-1412.

Yoshiba, Y., Kiyosue, T., Katagiri, T., Ueda, H., Mizoguchi, T., Yamaguchi-Shinozaki, K., et al. (1995). Correlation between the induction of a gene for $\Delta 1$-pyrroline-5-carboxylate synthetase and the accumulation of proline in Arabidopsis thaliana under osmotic stress. Plant J. 7, 751-760.

Yoshiba, Y., Kiyosue, T., Nakashima, K., Yamaguchi-shinozaki, K., and Shinozaki, K. (1997). Regulation of levels of proline as an osmolyte in plants under water stress. Plant Cell Physiol. 38, 1095-1102.

Zhai, H., Bai, X., Zhu, Y., Li, Y., Cai, H., Ji, W., et al. (2010). A singlerepeat R3-MYB transcription factor MYBC1 negatively regulates freezing tolerance in Arabidopsis. Biochem. Biophys. Res. Commun. 394, 1018-1023. doi: 10.1016/j.bbrc.2010.03.114

Zhang, J.-Y., Broeckling, C. D., Blancaflor, E. B., Sledge, M. K., Sumner, L. W., and Wang, Z.-Y. (2005). Overexpression of WXP1, a putative Medicago truncatula AP2 domain-containing transcription factor gene, increases cuticular wax accumulation and enhances drought tolerance in transgenic alfalfa (Medicago sativa). Plant J. 42, 689-707. doi: 10.1111/j.1365-313X.2005. 02405.x

Zhang, J. Y., Broeckling, C. D., Sumner, L. W., and Wang, Z. Y. (2007). Heterologous expression of two Medicago truncatula putative ERF transcription factor genes, WXP1 and WXP2, in Arabidopsis led to increased leaf wax accumulation and improved drought tolerance, but differential response in freezing tolerance. Plant Mol. Biol. 64, 265-278. doi: 10.1007/511103-007-9150-2

Zhang, L.-L., Zhao, M.-G., Tian, Q.-Y., and Zhang, W.-H. (2011). Comparative studies on tolerance of Medicago truncatula and Medicago falcata to freezing. Planta 234, 445-457. doi: 10.1007/s00425-011-1416-x

Zhu, J., Verslues, P. E., Zheng, X., Lee, B., Zhan, X., Manabe, Y., et al. (2005). HOS10 encodes an R2R3-type MYB transcription factor essential for cold acclimation in plants. Proc. Natl. Acad. Sci. U.S.A. 102, 9966-9971. doi: 10.1073/pnas.0503960102

Zou, H., Wu, Z., Yang, Q., Zhang, X., Cao, M., Jia, W., et al. (2006). Gene expression analyses of ZmPtil, encoding a maize Pti-like kinase, suggest a role in stress signaling. Plant Sci. 171, 99-105. doi: 10.1016/j.plantsci.2006.02.017

Conflict of Interest Statement: The authors declare that the research was conducted in the absence of any commercial or financial relationships that could be construed as a potential conflict of interest.

Copyright (c) 2016 Calzadilla, Maiale, Ruiz and Escaray. This is an open-access article distributed under the terms of the Creative Commons Attribution License (CC BY). The use, distribution or reproduction in other forums is permitted, provided the original author(s) or licensor are credited and that the original publication in this journal is cited, in accordance with accepted academic practice. No use, distribution or reproduction is permitted which does not comply with these terms. 